\title{
Sufficient Conditions for a Graph to be Super Restricted Edge-Connected
}

\author{
Shiying Wang and Shangwei Lin \\ School of Mathematical Sciences, Shanxi University, Taiyuan 030006, People's Republic of China
}

\begin{abstract}
Restricted edge connectivity is a more refined network reliability index than edge connectivity. A restricted edge cut $F$ of a connected graph $G$ is an edge cut such that $G-F$ has no isolated vertex. The restricted edge connectivity $\lambda^{\prime}$ is the minimum cardinality over all restricted edge cuts. We call $G \lambda^{\prime}$-optimal if $\lambda^{\prime}=\xi$, where $\xi$ is the minimum edge degree in $G$. Moreover, a $\lambda^{\prime}$-optimal graph $G$ is called a super restricted edge-connected graph if every minimum restricted edge cut separates exactly one edge. Let $D$ and $g$ denote the diameter and girth of $G$, respectively. In this paper, we first present a necessary condition for non-super restricted edge-connected graphs with minimum degree $\delta \geq 3$ and $D \leq g-2$. Next, we prove that a connected graph with minimum degree $\delta \geq 3$ and $D \leq g-3$ is super restricted edge-connected. Finally, we give some sufficient conditions on the conditional diameter and the girth for super restricted edge-connected graphs. () 2007 Wiley Periodicals, Inc. NETWORKS, Vol. 51(3), 200-209 2008
\end{abstract}

Keywords: restricted edge connectivity; super restricted edgeconnected graphs; diameter; girth

\section{TERMINOLOGY AND INTRODUCTION}

A processor interconnection network or a communications network is conveniently modeled by an undirected graph $G=(V, E)$, in which the vertex set $V$ corresponds to processors or switching elements, and the edge set $E$ corresponds to communication links. One fundamental consideration in the design of networks is reliability. When studying network reliability, one often considers a network model [9] whose vertices are perfectly reliable while edges may fail independently with the same probability $\rho \in(0,1)$. For subsets $A$ and $A^{\prime}$ of $V$, we denote by $\left[A, A^{\prime}\right]$ the set of edges with one end in $A$ and the other in $A^{\prime}$. An edge cut of $G$ is a subset of $E$ of the

Received February 2006; accepted June 2007

Correspondence to: Dr. S. Wang; e-mail: shiying@sxu.edu.cn

Contract grant sponsor: Shanxi Province Science Foundation; Contract grant number: 20041002

Contract grant sponsor: China National Science Foundation; Contract grant number: 10471081

DOI 10.1002/net.20217

Published online 14 December 2007 in Wiley InterScience (www. interscience.wiley.com).

C 2007 Wiley Periodicals, Inc. form $[A, \bar{A}]$, where $A$ is a nonempty proper subset of $V$ and $\bar{A}=V-A$. The edge connectivity $\lambda=\lambda(G)$ is the minimum size of any edge cut of $G$. Let $m_{i}$ be the number of edge cuts of size $i$. Then the probability of $G$ being connected is

$$
R(G ; \rho)=1-\sum_{i=\lambda}^{\varepsilon} m_{i} \rho^{i}(1-\rho)^{\varepsilon-i},
$$

where $\varepsilon=|E(G)|$. The polynomial $R(G ; \rho)$ is called the (all-terminal) reliability of $G$. Clearly, the larger $R(G ; \rho)$ is, the more reliable is the network. But in general, to determine $R(G ; \rho)$, i.e., to determine every $m_{i}$, is $N P$-hard $[5,16]$. When $\rho$ is sufficiently small, the maximum of $R(G ; \rho)$ can be obtained by maximizing $\lambda$ first and then minimizing $m_{\lambda}, m_{\lambda+1}, \ldots, m_{\varepsilon}$ sequentially [18].

The degree $d(v)$ of the vertex $v$ in $G$ is the number of vertices adjacent to $v$, and the edge degree $d(e)$ of the edge $e=u v$ in $G$ is $d(u)+d(v)-2$. Let $\delta=\delta(G)$ and $\xi=$ $\xi(G)$ denote the minimum degree and the minimum edge degree in $G$, respectively. It is well known that $\lambda \leq \delta$ for a general graph. If $\lambda=\delta$, then $G$ is said to be maximally edge-connected. To minimize $m_{\lambda}$, Bauer et al. [6,7] defined the super- $\lambda$ graphs. A graph $G$ is said to be super $-\lambda$ if each of its minimum edge cuts isolates a vertex. That is, if $F$ is a set of $\lambda$ edges such that $G-F$ is disconnected, then $F$ is the set of edges incident with a certain vertex of $G$. If $G$ is super $-\lambda$, then $\lambda=\delta$. But the converse is not true. As a more refined index than edge connectivity, restricted edge connectivity was proposed by Esfahanian and Hakimi [9]. A set of edges $F$ in a connected graph $G$ is called a restricted edge cut if $G-F$ is disconnected and contains no isolated vertex. If such an edge cut exists, then the restricted edge connectivity of $G$, denoted by $\lambda^{\prime}=\lambda^{\prime}(G)$, is defined to be the minimum number of edges over all restricted edge cuts of $G$. A restricted edge cut $F$ is called a $\lambda^{\prime}$-cut if $|F|=\lambda^{\prime}(G)$. A connected graph $G$ is called $\lambda^{\prime}$-connected if $\lambda^{\prime}(G)$ exists. Esfahanian and Hakimi [9] showed that each connected graph $G$ of order $v \geq 4$ except a star $K_{1, v-1}$ is $\lambda^{\prime}$-connected and satisfies $\lambda(G) \leq \lambda^{\prime}(G) \leq \xi(G)$. So a connected graph $G$ must be $\lambda^{\prime}$-connected if $\delta \geq 3$. A graph $G$ is called a $\lambda^{\prime}$-optimal graph if $\lambda^{\prime}(G)=\xi(G)$. Moreover, $G$ is super restricted edgeconnected, in short, super $-\lambda^{\prime}$, if every minimum restricted 
edge cut of $G$ isolates one edge. That is, every minimum restricted edge cut of $G$ is a set of edges adjacent to a certain edge with minimum edge degree in $G$. By definition, a super$\lambda^{\prime}$ graph must be a $\lambda^{\prime}$-optimal graph. However, the converse is not true because there are many $\lambda^{\prime}$-optimal graphs that are not super $-\lambda^{\prime}$. A trivial example is $C_{\nu}(\nu \geq 6)$, the cycle of length $v$. It should be pointed out that if $\delta \geq 3$, then a $\lambda^{\prime}$ optimal graph must be super $-\lambda$. In fact, a graph $G$ is super $-\lambda$ if and only if $\lambda=\delta<\lambda^{\prime}[14,15]$.

For $u, v \in V(G)$, the distance $d(u, v)=d_{G}(u, v)$ between them is the length of a shortest path from $u$ to $v$. The diameter $D=D(G)$ is the maximum distance between two vertices of $G$ and the girth $g=g(G)$ is the length of a shortest cycle in $G$. Some sufficient conditions for a graph to be maximally edge-connected or super $-\lambda$ have been given in terms of its diameter $D$ and its girth $g$.

\section{Theorem A.}

(1) [17]. $G$ is maximally edge-connected if $D \leq 2\lfloor(g-$ 1) $/ 2$.

(2) [10]. $G$ is super $-\lambda$ if $\delta \geq 3$ and $D \leq 2\lfloor(g-1) / 2\rfloor-1$.

(3) [2]. $G$ is super $-\lambda$ if $G$ is a bipartite graph with $\delta \geq 3$ and $D \leq g-2$.

(4) $[11] \cdot \lambda^{\prime}(G) \geq 2 \delta-2$ if $\delta \geq 3$ and $D \leq 2\lfloor(g-1) / 2\rfloor-1$.

Later, the following theorem was obtained in [19], which generalized the above-mentioned results.

Theorem B ([19]). If $\delta \geq 3$ and $D \leq g-2$, then $G$ is $\lambda^{\prime}$-optimal, that is, $\lambda^{\prime}(G)=\xi(G)$.

Recently, many sufficient conditions for a graph to be $\lambda^{\prime}$ optimal were given with girth $g$ and diameter $D[4,13,14]$. In particular, a theorem given in [4] improves Theorem B.

Theorem $\mathbf{B}^{\prime}([4])$. Let $G$ be a $\lambda^{\prime}$-connected graph with $\delta \geq$ 2. Then $G$ is $\lambda^{\prime}$-optimal if $D \leq g-2$.

Wang and $\mathrm{Li}$ [19] pointed out that the graph $W$ shown in Figure 1 satisfying the conditions in Theorem B is not super $-\lambda^{\prime}$, and made the following conjecture.

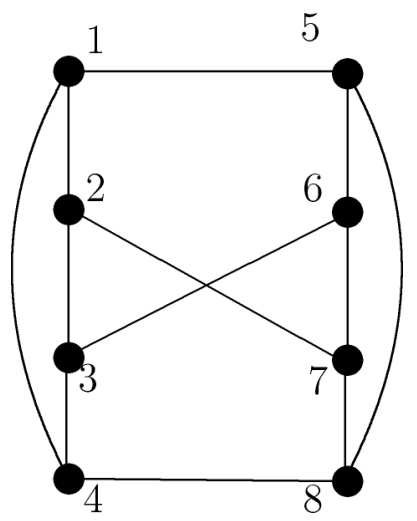

FIG. 1. A non-super- $\lambda^{\prime}$ graph $W$ with $\delta=3, D=2$, and $g=4$.

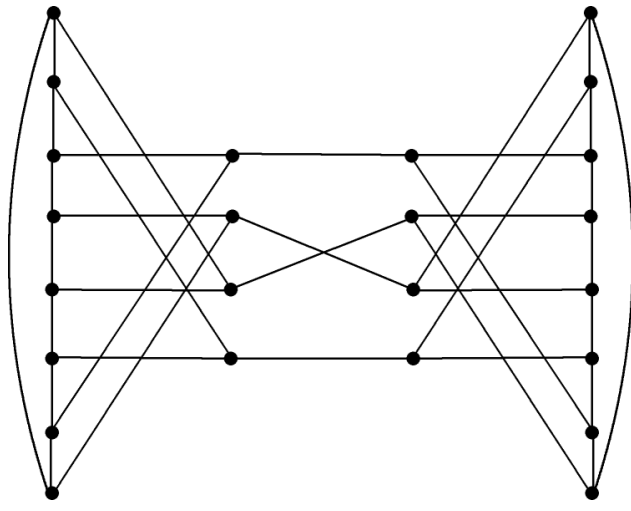

FIG. 2. A non-super $-\lambda^{\prime}$ graph with $\delta=3, D=4$, and $g=6$.

Conjecture A ([19]). Suppose that $G$ is not isomorphic to $W$ shown in Figure 1 . If $\delta \geq 3$ and $D \leq g-2$, then $G$ is super- $\lambda^{\prime}$.

In fact, this conjecture is false. A counterexamples is given in Figure 2. In this paper, we shall present a necessary condition for non-super $-\lambda^{\prime}$ graphs with $\delta \geq 3$ and $D \leq g-2$. According to this necessary condition, it can be easily seen that a graph $G$ satisfying $\delta \geq 3$ and $D \leq g-3$ is super $-\lambda^{\prime}$.

For $A, B \subseteq V$, let $d(A, B)=d_{G}(A, B)=\min \{d(u, v)$ : $u \in A, v \in B\}$. In particular, if $A=\{u\}$, then we denote $d(A, B)$ by $d(u, B)$. For $U \subseteq V$, let $G[U]$ be the subgraph induced by $U$. Sometimes, we also regard $d(A, B)$ as the distance between $G[A]$ and $G[B]$. In [3], some conditions were given to guarantee $\lambda^{\prime}=\xi$ for graphs with diameter $D \geq g-1$ by introducing the concept of conditional diameter. For any property $P$ satisfied by some pairs $\left(V_{1}, V_{2}\right)$ of non-empty subsets of $V$, the conditional diameter, or simply the $P$-diameter of $G$, is defined by (see [1])

$\operatorname{Diam}_{P}=\max \left\{d\left(V_{1}, V_{2}\right): V_{1}, V_{2} \subseteq V,\left(V_{1}, V_{2}\right)\right.$ satisfies $\left.P\right\}$.

In particular, for any non-negative integer $k$, let

$$
\operatorname{Diam}_{k}=\max \left\{d\left(V_{1}, V_{2}\right): \delta\left(G\left[V_{i}\right]\right) \geq k, i=1,2\right\} .
$$

Notice that $D=$ Diam $_{0} \geq \operatorname{Diam}_{1} \geq \cdots \geq$ Diam $_{\delta}$. Clearly, $D_{\text {Diam }}$ is the maximum distance between two edges of $G$, and Diam $_{2}$ is the maximum distance between two cycles of $G$.

Theorem $\mathbf{C}$ ([3]). Let $G$ be a $\lambda^{\prime}$-connected graph with $\delta \geq$ 2. Then $G$ is a $\lambda^{\prime}$-optimal graph if Diam $1 \leq g-3$ or both Diam $_{1}=g-2$ and Diam $2 \leq g-5$, for odd girth $g \geq 5$; and if both Diam $1 \leq g-3$ and Diam $2 \leq g-4$, for even girth $g \geq 4$.

In this paper, it is shown that the conditions in Theorem $\mathrm{C}$ also guarantee that $G$ is super $-\lambda^{\prime}$ if $\delta \geq 3$ instead of $\delta \geq 2$. Considering $C_{v}(v \geq 6)$, we know that the condition $\delta \geq 3$ is necessary.

For graph-theoretical terminology and notation, not defined here, we follow [8]. We only consider finite, undirected and simple connected graphs $G=(V, E)$. For every 
$A \subseteq V$ and every non-negative integer $k, N^{k}(A)=N_{G}^{k}(A)=$ $\{w \in V: d(w, A)=k\}$ denotes the neighborhood of $A$ at distance $k$. Let $G^{\prime}=\left(V^{\prime}, E^{\prime}\right)$ be a subgraph of $G$. We define $N_{G^{\prime}}^{k}(A)=N_{G}^{k}(A) \cap V^{\prime}$. Observe that $N_{G}^{0}(A)=A$. When $k=1$, we simply use $N_{G}(A)$ instead of $N_{G}^{1}(A)$. Let $F \subseteq E$ and $x \in V$. We denote by $F(x)$ the subset of $F$ whose edges are incident with $x$. For $A \subseteq V$, let $F(A)=\cup_{x \in A} F(x)$.

\section{PROPERTIES OF $\lambda^{\prime}$-OPTIMAL GRAPHS}

Let $G$ be a $\lambda^{\prime}$-connected graph and let $F$ be an arbitrary $\lambda^{\prime}$-cut of $G$. By the minimality of $F$, the graph $G-F$ consists of exactly two components, say $G_{1}$ and $G_{2}$. Then, $F=\left[V\left(G_{1}\right), V\left(G_{2}\right)\right]$. As there exist $X^{0} \subseteq V\left(G_{1}\right)$ and $Y^{0} \subseteq V\left(G_{2}\right)$ such that both $X^{0}$ and $Y^{0}$ are the end vertices of the edges of $F$, we will also write $F=\left[X^{0}, Y^{0}\right]$. Let $G_{1}^{0}=G_{1}\left[X^{0}\right], G_{2}^{0}=G_{2}\left[Y^{0}\right]$ and let

$m=\max \left\{d\left(x, X^{0}\right): x \in V\left(G_{1}\right)\right\}$,

$$
X^{i}=N_{G_{1}}^{i}\left(X^{0}\right), G_{1}^{i}=G\left[X^{i}\right], \quad i=1, \ldots, m ;
$$

$n=\max \left\{d\left(y, Y^{0}\right): y \in V\left(G_{2}\right)\right\}$,

$$
Y^{i}=N_{G_{2}}^{i}\left(Y^{0}\right), G_{2}^{i}=G\left[Y^{i}\right], \quad i=1, \ldots, n .
$$

It is easy to see that $X^{i} \cap X^{j}=\varnothing, Y^{i} \cap Y^{j}=\emptyset$ if $i \neq j$, and $V\left(G_{1}\right)=\cup_{i=0}^{m} X^{i}, V\left(G_{2}\right)=\cup_{i=0}^{n} Y^{i}$. (We will use such notation in this section and next section.)

The main goal of this section is to give some useful properties of $G_{1}$ and $G_{2}$. By reason of symmetry, we only discuss $G_{1}$. We first present the structure of $G_{1}$ when $m=0$.

Lemma 2.1. Let $G$ be a $\lambda^{\prime}$-optimal graph with $\delta \geq 3, g \geq 4$. If $F=\left[X^{0}, Y^{0}\right]$ is a $\lambda^{\prime}$-cut such that $\left|V\left(G_{1}\right)\right|=\left|X^{0}\right| \geq 3$, then $|F(w)|=1$ for any $w \in V\left(G_{1}\right)$ and $G_{1}$ is a complete bipartite graph $K_{p, q}$, where $p \geq 2, q \geq 2$.

Proof. Since $V\left(G_{1}\right)=X^{0}$, it follows that $|F(x)| \geq 1$ for any $x \in V\left(G_{1}\right)$. Consider any edge $e=x y \in E\left(G_{1}\right)$. Since $g \geq 4$, we have $N_{G_{1}}(x) \cap N_{G_{1}}(y)=\emptyset, E\left(G_{1}\left[N_{G_{1}}(x)\right]\right)=\emptyset$ and $E\left(G_{1}\left[N_{G_{1}}(y)\right]\right)=\emptyset$. Therefore, we have

$$
\begin{aligned}
\xi & \leq d(e) \\
& =d(x)+d(y)-2 \\
& =|F(x)|+|F(y)|+\left|N_{G_{1}}(x)-\{y\}\right|+\left|N_{G_{1}}(y)-\{x\}\right| \\
& =|F(x)|+|F(y)|+\left|N_{G_{1}}(x) \cup N_{G_{1}}(y)-\{x, y\}\right| \\
& \leq|F(x)|+|F(y)|+\left|F\left(N_{G_{1}}(x) \cup N_{G_{1}}(y)-\{x, y\}\right)\right| \\
& =\left|F\left(N_{G_{1}}(x) \cup N_{G_{1}}(y)\right)\right| \leq\left|F\left(V\left(G_{1}\right)\right)\right|=|F|=\lambda^{\prime}=\xi .
\end{aligned}
$$

This implies that $V\left(G_{1}\right)=N_{G_{1}}(x) \cup N_{G_{1}}(y)$ and $|F(u)|=1$ for any $u \in V\left(G_{1}\right)-\{x, y\}$.

Since $\left|V\left(G_{1}\right)\right| \geq 3$, we may assume, without loss of generality, $N_{G_{1}}(x)-\{y\} \neq \emptyset$. Picking arbitrarily $y^{\prime} \in N_{G_{1}}(x)-\{y\}$ and considering the edge $x y^{\prime}$ similarly, we have $V\left(G_{1}\right)=$ $N_{G_{1}}(x) \cup N_{G_{1}}\left(y^{\prime}\right), N_{G_{1}}(x) \cap N_{G_{1}}\left(y^{\prime}\right)=\emptyset$, and $|F(u)|=1$ for any $u \in V\left(G_{1}\right)-\left\{x, y^{\prime}\right\}$. Hence, $N_{G_{1}}(y)=N_{G_{1}}\left(y^{\prime}\right)$ and $|F(y)|=1$. Since $|F(y)|=1$ and $\delta \geq 3$, we have $N_{G_{1}}(y)-\{x\} \neq \emptyset$. Considering $x^{\prime} y$ for any $x^{\prime} \in N_{G_{1}}(y)-\{x\}$ similarly, we conclude that $N_{G_{1}}(x)=N_{G_{1}}\left(x^{\prime}\right)$ and $|F(x)|=$ 1. Thus $|F(u)|=1$ for any $u \in V\left(G_{1}\right), G_{1}$ is a complete bipartite graph with bipartition $\left(N_{G_{1}}(x), N_{G_{1}}(y)\right)$, and $\left|N_{G_{1}}(x)\right| \geq 2,\left|N_{G_{1}}(y)\right| \geq 2$.

Lemma 2.2. Let $G$ be a $\lambda^{\prime}$-optimal graph with $\delta \geq 3$. If $F=\left[X^{0}, Y^{0}\right]$ is a $\lambda^{\prime}$-cut such that $1 \leq m \leq(g-3) / 2$, then $E\left(G_{1}^{m}\right) \neq \emptyset$.

Proof. Suppose, on the contrary, $E\left(G_{1}^{m}\right)=\emptyset$. Then $N_{G}(u) \subseteq X^{m-1}$ for any $u \in X^{m}$. For any edge $e=x y \in$ $\left[X^{m}, X^{m-1}\right]$, where $x \in X^{m}, y \in X^{m-1}$, let $A_{m}=N_{G_{1}^{m}}(y)-$ $\{x\}, A_{m-1}=N_{G_{1}^{m-1}}\left(A_{m}\right)-\{y\}, B_{m-1}=N_{G}(x)-\{y\}, C_{m-1}=$ $N_{G_{1}^{m-1}}(y)$, and let $A_{0}=N_{G_{1}^{0}}^{m-1}\left(A_{m-1}\right), B_{0}=N_{G_{1}^{0}}^{m-1}\left(B_{m-1}\right)$, $C_{0}=N_{G_{1}^{0}}^{m-1}\left(C_{m-1}\right), D_{0}=N_{G_{1}^{0}}^{m-1}(y)$. Clearly, if $m=1$, then $D_{0}=\{y\}$. Since $m \leq(g-3) / 2$, it follows that $g \geq 2 m+3$. This implies the following: (1) $A_{0}, B_{0}, C_{0}$, and $D_{0}$ are pairwise disjoint subsets of $X^{0}$; (2) $\left|A_{0}\right| \geq\left|A_{m-1}\right| \geq\left|A_{m}\right|$, $\left|B_{0}\right| \geq\left|B_{m-1}\right|$, and $\left|C_{0}\right| \geq\left|C_{m-1}\right|$; (3) $\left|D_{0}\right| \geq\left|N_{G_{1}^{m-2}}(y)\right|$ if $m \geq 2$. Therefore, we have

$$
\begin{aligned}
\xi \leq & d(e) \\
& =\left|A_{m}\right|+\left|B_{m-1}\right|+\left|C_{m-1}\right|+ \begin{cases}|F(y)| & \text { if } m=1 \\
\left|N_{G_{1}^{m-2}}(y)\right| & \text { if } m \geq 2\end{cases} \\
& \leq\left|A_{0}\right|+\left|B_{0}\right|+\left|C_{0}\right|+ \begin{cases}|F(y)| & \text { if } m=1 \\
\left|D_{0}\right| & \text { if } m \geq 2\end{cases} \\
& \leq\left|F\left(A_{0}\right)\right|+\left|F\left(B_{0}\right)\right|+\left|F\left(C_{0}\right)\right|+\left|F\left(D_{0}\right)\right| \\
& \leq\left|F\left(X^{0}\right)\right|=|F|=\lambda^{\prime}=\xi .
\end{aligned}
$$

This implies $\left|A_{0}\right|=\left|A_{m}\right|$ and hence $\left|A_{m-1}\right|=\left|A_{m}\right|$. Suppose $A_{m} \neq \emptyset$ and let $x^{\prime} \in A_{m}$. By assumption, $N_{G_{1}^{m}}\left(x^{\prime}\right)=\emptyset$. Since $\left|A_{m-1}\right|=\left|A_{m}\right|$ and $g \geq 2 m+3$, we conclude that $\mid N_{G_{1}^{m-1}}\left(x^{\prime}\right)-$ $\{y\} \mid=1$. Therefore, $d\left(x^{\prime}\right)=2$, which contradicts $\delta \geq 3$. So $A_{m}=\emptyset$, and hence $A_{m-1}=A_{0}=\emptyset$. Combining this with the equality $\left|F\left(A_{0}\right)\right|+\left|F\left(B_{0}\right)\right|+\left|F\left(C_{0}\right)\right|+\left|F\left(D_{0}\right)\right|=\left|F\left(X^{0}\right)\right|$, we have

$$
X^{0}=B_{0} \cup C_{0} \cup D_{0}=N_{G_{1}^{0}}^{m}(x) \cup C_{0} .
$$

Since $d(x) \geq \delta \geq 3$, it follows that $B_{m-1} \neq \emptyset$. If $m=1$, since $\left|F\left(B_{0}\right)\right|=\left|B_{0}\right|$, we have $\left|N_{G_{2}^{0}}(u)\right|=1$ for any $u \in B_{0}$. If $m \geq 2$, since $\left|B_{0}\right|=\left|B_{m-1}\right|$ and $g \geq 2 m+3$, we have $\left|N_{G_{1}^{m-2}}(u)\right|=1$ for any $u \in B_{m-1}$.

For any vertex $y^{\prime} \in B_{m-1}$, let $B_{m-1}^{\prime}=N(x)-\left\{y^{\prime}\right\}, C_{m-1}^{\prime}=$ $N_{G_{1}^{m-1}}\left(y^{\prime}\right)$, and $C_{0}^{\prime}=N_{G_{1}^{0}}^{m-1}\left(C_{m-1}^{\prime}\right)$. Considering the edge $x y^{\prime}$ similarly, we have $X^{0}=N_{G_{1}^{0}}^{m}(x) \cup C_{0}^{\prime},\left|N_{G_{2}^{0}}(v)\right|=1$ if $m=1$ and $\left|N_{G_{1}^{m-2}}(v)\right|=1$ if $m \geq 2$ for any $v \in B_{m-1}^{\prime}$. In particular, $\left|N_{G_{2}^{0}}(y)\right|=1$ if $m=1$ and $\left|N_{G_{1}^{m-2}}(y)\right|=1$ if $m \geq 2$ because $y \in B_{m-1}^{\prime}$. Combining this with $\delta \geq 3$, we 
have $C_{m-1} \neq \emptyset$ and hence $C_{0} \neq \emptyset$. It follows that $C_{0}=C_{0}^{\prime}$ from $N_{G_{1}^{0}}^{m}(x) \cup C_{0}=X^{0}=N_{G_{1}^{0}}^{m}(x) \cup C_{0}^{\prime}$. Therefore, there exists a cycle of length at most $2 m+2$ through $y, x, y^{\prime}$, which contradicts $g \geq 2 m+3$. Therefore, $E\left(G_{1}^{m}\right) \neq \emptyset$.

Lemma 2.3. Let $G$ be a $\lambda^{\prime}$-optimal graph with $\delta \geq 3$ and let $F=\left[X^{0}, Y^{0}\right]$ be a $\lambda^{\prime}$-cut such that $1 \leq m=(g-3) / 2$. Then the graph $G_{1}\left[X^{m} \cup X^{m-1}\right]$ contains cycles.

Proof. Let $T=G_{1}\left[X^{m} \cup N_{G_{1}^{m-1}}\left(X^{m}\right)\right]$. Clearly, $d_{T}(w) \geq$ 1 for any vertex $w$ of $T$, and if $d_{T}(w)=1$ then $w \in$ $N_{G_{1}^{m-1}}\left(X^{m}\right)$. Let $\Omega=\left\{w \in V(T): d_{T}(w)=1\right\}$. Then $\Omega \subseteq N_{G_{1}^{m-1}}\left(X^{m}\right)$. Notice that $N_{G_{1}^{m-1}}(\Omega) \subseteq X^{m-1}-N_{G_{1}^{m-1}}\left(X^{m}\right)$ and let us consider the subgraph $H=T-\Omega$. If each vertex $x \in V(H)$ satisfies $d_{H}(x) \geq 2$, then there must be a cycle in $H$ and hence in $G_{1}\left[X^{m} \cup X^{m-1}\right]$. Thus, it is enough to show that $\delta(H) \geq 2$.

Suppose, on the contrary, there exists a vertex $x \in V(H)$ such that $d_{H}(x) \leq 1$. Obviously, $x \in X^{m}$ and there are at least $d_{G}(x)-1 \geq 2$ vertices adjacent to $x$ in $\Omega$. Let $y, z$ be distinct vertices in $N(x) \cap \Omega$. By the definition of $\Omega$, we know $N_{G_{1}^{m}}(y)-\{x\}=N_{G_{1}^{m}}(z)-\{x\}=\emptyset$. Let $A_{m}=N_{G_{1}^{m}}(x), B_{m-1}=N_{G_{1}^{m-1}}(x)-\{y\}, C_{m-1}=$ $N_{G_{1}^{m-1}}(y)$, and let $A_{0}=N_{G_{1}^{0}}^{m}\left(A_{m}\right), B_{0}=N_{G_{1}^{0}}^{m-1}\left(B_{m-1}\right)$, $C_{0}=N_{G_{1}^{0}}^{m-1}\left(C_{m-1}\right), D_{0}=N_{G_{1}^{0}}^{m-1}(y)$. Clearly, $z \in B_{m-1}$. Since $m=(g-3) / 2$, that is, $g=2 m+3$, it follows that $A_{0}, B_{0}, C_{0}, D_{0}$ are pairwise disjoint and $\left|A_{m}\right| \leq$ $\left|A_{0}\right|,\left|B_{m-1}\right| \leq\left|B_{0}\right|,\left|C_{m-1}\right| \leq\left|C_{0}\right|$. Hence $\xi \leq d(x y) \leq$ $\left|A_{0}\right|+\left|B_{0}\right|+\left|C_{0}\right|+\left|F\left(D_{0}\right)\right| \leq\left|F\left(A_{0}\right)\right|+\left|F\left(B_{0}\right)\right|+$ $\left|F\left(C_{0}\right)\right|+\left|F\left(D_{0}\right)\right| \leq\left|F\left(X^{0}\right)\right|=\lambda^{\prime}=\xi$. This implies $X^{0}=A_{0} \cup B_{0} \cup C_{0} \cup D_{0}, d_{G_{1}^{m-2}}(z)=1$ if $m \geq 2$ and $d_{G_{2}^{0}}(z)=1$ if $m=1$. It follows that $N_{G_{1}^{m-1}}(z) \neq \emptyset$ from $d_{G}(z) \geq 3$. On the other hand, $N_{G_{1}^{0}}^{m-1}\left(N_{G_{1}^{m-1}}(z)\right) \subseteq X^{0}=A_{0} \cup B_{0} \cup C_{0} \cup D_{0}$. This implies that there exists a cycle of length at most $2 m+2$ through $x, z$, a contradiction. The proof is complete.

Lemmas 2.2 and 2.3 show that $G_{1}^{m}$ must contain edges and $G_{1}\left[X^{m} \cup X^{m-1}\right]$ must contain cycles when $m=(g-3) / 2$. It will be proved that either $G_{1}^{m}$ contains edges or $G_{1}\left[X^{m} \cup\right.$ $\left.X^{m-1} \cup X^{m-2}\right]$ contains cycles when $m=(g-1) / 2$ as follows.

Lemma 2.4. Let $G$ be a $\lambda^{\prime}$-optimal graph with $\delta \geq 3$ and let $F=\left[X^{0}, Y^{0}\right]$ be a $\lambda^{\prime}$-cut such that $2 \leq m=(g-1) / 2$. If $G_{1}^{m}$ contains no edge, then $G_{1}\left[X^{m} \cup X^{m-1} \cup X^{m-2}\right]$ contains cycles.

Proof. Let $T=G_{1}\left[X^{m} \cup X^{m-1} \cup N_{G_{1}^{m-2}}\left(X^{m-1}\right)\right]$. Clearly, $d_{T}(w) \geq 1$ for any vertex $w$ of $T$, and if $d_{T}(w)=1$ then $w \in$ $N_{G_{1}^{m-2}}\left(X^{m-1}\right)$. Let $\Omega=\left\{w \in V(T): d_{T}(w)=1\right\}$. Then $\Omega \subseteq$ $N_{G_{1}^{m-2}}\left(X^{m-1}\right)$. Notice that $N_{G_{1}^{m-2}}(\Omega) \subseteq X^{m-2}-N_{G_{1}^{m-2}}\left(X^{m-1}\right)$ and let us consider the subgraph $H=T-\Omega$. If $\delta(H) \geq 2$, we are done. Otherwise, set $\Psi=\left\{w \in V(H): d_{H}(w) \leq 1\right\}$. It is easy to see that $\Psi \subseteq X^{m-1}$. Let $x \in \Psi$. Then $\left|N_{G_{1}^{m-2}}(x) \cap \Omega\right| \geq$ $d_{G}(x)-1 \geq 2$. Pick two distinct vertices $y_{1}, y_{2} \in N(x) \cap \Omega$. By the definition of $\Omega, N_{G_{1}^{m-1}}\left(y_{1}\right)-\{x\}=N_{G_{1}^{m-1}}\left(y_{2}\right)-\{x\}=\emptyset$.
Let $B_{m-2}=N_{G_{1}^{m-2}}(x)-\left\{y_{1}\right\}, C_{m-2}=N_{G_{1}^{m-2}}\left(y_{1}\right)$, and let $B_{0}=N_{G_{1}^{0}}^{m-2}\left(B_{m-2}\right), C_{0}=N_{G_{1}^{0}}^{m-2}\left(C_{m-2}\right), D_{0}=N_{G_{1}^{0}}^{m-2}\left(y_{1}\right)$. Clearly, $y_{2} \in B_{m-2}$. Since $g=2 m+1$, we have: (1) $B_{0}, C_{0}$, and $D_{0}$ are pairwise disjoint; (2) $\left|B_{0}\right| \geq\left|B_{m-2}\right|$ and $\left|C_{0}\right| \geq$ $\left|C_{m-2}\right|$; (3) $\left|D_{0}\right| \geq\left|N_{G_{1}^{m-3}}\left(y_{1}\right)\right|$ if $m \geq 3$.

Claim 1. $\left|N_{G_{1}^{m-2}}(x)\right|=d_{G}(x)-1$.

Since $\left|N_{G_{1}^{m-2}}(x) \cap \Omega\right| \geq d_{G}(x)-1$, we have $d_{G}(x)-$ $1 \leq\left|N_{G_{1}^{m-2}}(x)\right| \leq d_{G}(x)$. Suppose $\left|N_{G_{1}^{m-2}}(x)\right|=d_{G}(x)$. Then $\xi \leq d\left(x y_{1}\right) \leq\left|B_{m-2}\right|+\left|C_{m-2}\right|+\left|F\left(D_{0}\right)\right| \leq\left|B_{0}\right|+\left|C_{0}\right|+$ $\left|F\left(D_{0}\right)\right| \leq\left|F\left(B_{0}\right)\right|+\left|F\left(C_{0}\right)\right|+\left|F\left(D_{0}\right)\right| \leq\left|F\left(X^{0}\right)\right|=\lambda^{\prime}=\xi$. This implies that $X^{0}=B_{0} \cup C_{0} \cup D_{0}, d_{G^{m-3}}\left(y_{2}\right)=1$ if $m \geq 3$ and $d_{G_{2}^{0}}\left(y_{2}\right)=1$ if $m=2$. Since $d\left(y_{2}\right) \geq 3$ and $N_{G_{1}^{m-1}}\left(y_{2}\right)-\{x\}=\emptyset$, there exists at least one vertex, say $w$, in $N_{G_{1}^{m-2}}\left(y_{2}\right)$. Noticing that $N_{G_{1}^{0}}^{m-2}(w) \subseteq X^{0}=B_{0} \cup C_{0} \cup D_{0}$, we can find a cycle of length at most $2 m$ through $y_{2}, w$, a contradiction completing the proof of Claim 1.

By Claim 1, we can assume $N_{G}(x)-N_{G_{1}^{m-2}}(x)=\{z\}$. Similarly, we have

$$
\begin{aligned}
\xi & \leq d\left(x y_{1}\right) \\
& =|\{z\}|+\left|B_{m-2}\right|+\left|C_{m-2}\right|+ \begin{cases}\left|F\left(y_{1}\right)\right| & \text { if } m=2 \\
\left|N_{G_{1}^{m-3}}\left(y_{1}\right)\right| & \text { if } m \geq 3\end{cases} \\
& \leq 1+\left|B_{0}\right|+\left|C_{0}\right|+ \begin{cases}\left|F\left(y_{1}\right)\right| & \text { if } m=2 \\
\left|D_{0}\right| & \text { if } m \geq 3\end{cases} \\
& \leq 1+\left|F\left(B_{0}\right)\right|+\left|F\left(C_{0}\right)\right|+\left|F\left(D_{0}\right)\right| \\
& \leq 1+\left|F\left(X^{0}\right)\right|=1+\lambda^{\prime}=1+\xi .
\end{aligned}
$$

Therefore, either $X^{0}=B_{0} \cup C_{0} \cup D_{0}$ or $\left|X^{0}\right|=\mid B_{0} \cup C_{0} \cup$ $D_{0} \mid+1$.

Claim 2. $\max \left\{\left|N_{G_{1}^{m-2}}(y)\right|: y \in N_{G_{1}^{m-2}}(x)\right\}>0$.

Suppose, on the contrary, that $N_{G_{1}^{m-2}}(y)=\emptyset$ for any vertex $y \in N_{G_{1}^{m-2}}(x)$. Then $C_{m-2}=\emptyset$ and hence $C_{0}=\emptyset$. Assume $X^{0}=B_{0} \cup D_{0}$. If $z \in X^{m}$, then since $E\left(G_{1}^{m}\right)=\emptyset$ and $\delta \geq 3$, there is a vertex $x^{\prime} \in N_{G_{1}^{m-1}}(z)-\{x\}$. Considering $N_{G_{1}^{0}}^{m-1}\left(x^{\prime}\right)$, we can find a cycle of length at most $2 m$ through $x^{\prime}, z, x$, a contradiction. If $z \in X^{m-1}$, then since $N_{G_{1}^{0}}^{m-1}(z) \subseteq X^{0}=B_{0} \cup$ $D_{0}$, we can find a cycle of length at most $2 m-1$ through $z, x$, a contradiction. Thus, $\left|X^{0}\right|=\left|B_{0}\right|+\left|D_{0}\right|+1$. This implies that $\xi \leq d\left(x y_{1}\right) \leq 1+\left|B_{m-2}\right|+\left|F\left(D_{0}\right)\right| \leq 1+\left|B_{0}\right|+\left|F\left(D_{0}\right)\right| \leq$ $1+\left|F\left(B_{0}\right)\right|+\left|F\left(D_{0}\right)\right|<1+\left|F\left(X^{0}\right)\right|=1+\xi$. Therefore, $\left|B_{m-2}\right|=\left|B_{0}\right|=\left|F\left(B_{0}\right)\right|$ and hence $\left|N_{G^{m-3}}\left(y_{2}\right)\right|=1$ if $m \geq 3$ and $\left|N_{G_{2}^{0}}\left(y_{2}\right)\right|=1$ if $m=2$. Combining this with $N_{G_{1}^{m-1}}\left(y_{2}\right)-\{x\}=\emptyset$ and $N_{G_{1}^{m-2}}\left(y_{2}\right)=\emptyset$, we have $d\left(y_{2}\right)=2$, a contradiction completing the proof of Claim 2 .

Claim 3. $G_{1}\left[X^{m} \cup X^{m-1} \cup X^{m-2}\right]$ contains cycles.

By Claim 1, we have $d_{G}(x)-1=\left|N_{G_{1}^{m-2}}(x)\right| \geq$ $\left|N_{G_{1}^{m-2}}(x) \cap \Omega\right| \geq d_{G}(x)-1$ and hence $N_{G_{1}^{m-2}}(x) \subseteq \Omega$. Thus, 
we can assume, without loss of generality, that $\left|N_{G_{1}^{m-2}}\left(y_{2}\right)\right|=$ $\max \left\{\left|N_{G_{1}^{m-2}}(y)\right|: y \in N_{G_{1}^{m-2}}(x)\right\}$ and pick $u \in N_{G_{1}^{m-2}\left(y_{2}\right)}$. Since $g=2 m+1$, we conclude that $N_{G_{1}^{0}}^{m-2}(u) \cap\left(B_{0} \cup C_{0} \cup\right.$ $\left.D_{0}\right)=\emptyset$. Therefore, $\left|X^{0}\right|=\left|B_{0}\right|+\left|C_{0}\right|+\left|D_{0}\right|+1$. Let $X^{0}-\left(B_{0} \cup C_{0} \cup D_{0}\right)=\{w\}$. Clearly, $N_{G_{1}^{0}}^{m-2}(u)=\{w\}$ and $N_{G_{1}^{m-2}}\left(y_{2}\right)=\{u\}$. If $z \in X^{m-1}$, then since $N_{G_{1}^{0}}^{m-1}(z) \subseteq X^{0}=$ $B_{0} \cup C_{0} \cup D_{0} \cup\{w\}$, there exists a cycle of length at most $2 m$, a contradiction implying $z \in X^{m}$. By the choice of $y_{2}$, we have $\left|C_{0}\right| \leq 1$. Since $d(z) \geq 3$ and $G_{1}^{m}$ contains no edge, there exist two distinct vertices, say $x_{1}, x_{2}$, in $N_{G_{1}^{m-1}}(z)-\{x\}$. Noticing $N_{G_{1}^{0}}^{m-1}\left(x_{i}\right) \cap\left(B_{0} \cup D_{0}\right)=\emptyset(i=1,2)$, we have $\left|C_{0}\right|=1, N_{G_{1}^{m-1}}(z)=\left\{x, x_{1}, x_{2}\right\},\left|N_{G_{1}^{0}}^{m-1}\left(x_{i}\right)\right|=1$ and hence $\left|N_{G_{1}^{m-2}}\left(x_{i}\right)\right|=1$. Since $\left|N_{G_{1}^{m-2}}(x) \cap \Omega\right| \geq d_{G}(x)-1 \geq 2$ for any $x \in \Psi$, we have $x_{1}, x_{2} \notin \Psi$. It follows that $d_{H-\Psi}(w) \geq 2$ for any vertex $w \in V(H)-\Psi$. Therefore, the subgraph $H-\Psi$ has cycles. The proof is complete.

Lemma 2.5. Let $G$ be a $\lambda^{\prime}$-optimal graph with $\delta \geq 3$ and let $F=\left[X^{0}, Y^{0}\right]$ be a $\lambda^{\prime}$-cut such that $1 \leq m=(g-2) / 2$. If $G_{1}^{m}$ contains no edge and $G_{1}\left[X^{m} \cup X^{m-1}\right]$ contains no cycle, then there exists a vertex $x$ in $X^{m}$ such that $\left|N_{G_{1}^{m-1}}(x)\right|=\left|N_{G_{1}^{0}}^{m}(x)\right|$ and $\left|N_{G_{1}^{0}}^{m-1}(y)\right|=\left|N_{G_{2}^{0}}^{m}(y)\right|=1$ for any $y \in N_{G}(x)$.

Proof. Let $T=G_{1}\left[X^{m} \cup N_{G_{1}^{m-1}}\left(X^{m}\right)\right]$. Clearly, $d_{T}(w) \geq$ 1 for any vertex $w$ of $T$, and if $d_{T}(w)=1$ then $w \in$ $N_{G_{1}^{m-1}}\left(X^{m}\right)$. Let $\Omega=\left\{w \in V(T): d_{T}(w)=1\right\}$. Then $\Omega \subseteq N_{G_{1}^{m-1}}\left(X^{m}\right)$. Notice that $N_{G_{1}^{m-1}}(\Omega) \subseteq X^{m-1}-N_{G_{1}^{m-1}}\left(X^{m}\right)$ and let us consider the subgraph $H=T-\Omega$. By assumption, $H$ has no cycle, which implies that there exists a vertex $x$ of $H$ such that $d_{H}(x) \leq 1$. Clearly, $x \in X^{m}$ and $\left|N_{G_{1}^{m-1}}(x) \cap \Omega\right| \geq d_{G}(x)-1 \geq 2$. Pick two distinct vertices $y_{1}, y_{2} \in N_{G_{1}^{m-1}}(x) \cap \Omega$. By the definition of $\Omega, N_{G_{1}^{m}}\left(y_{1}\right)-\{x\}$ $=N_{G_{1}^{m}}\left(y_{2}\right)-\{x\}=\emptyset$. Let $B_{m-1}=N_{G_{1}^{m-1}}(x)-\left\{y_{1}\right\}, C_{m-1}=$ $N_{G_{1}^{m-1}}\left(y_{1}\right), B_{0}=N_{G_{1}^{0}}^{m-1}\left(B_{m-1}\right), C_{0}=N_{G_{1}^{0}}^{m-1}\left(C_{m-1}\right), D_{0}=$ $N_{G_{1}^{0}}^{m-1}\left(y_{1}\right)$.

Considering the edge $x y_{1}$, we have $\xi \leq d\left(x y_{1}\right) \leq\left|B_{m-1}\right|+$ $\left|C_{m-1}\right|+\left|F\left(D_{0}\right)\right| \leq\left|B_{0}\right|+\left|C_{0}\right|+\left|F\left(D_{0}\right)\right| \leq\left|F\left(B_{0}\right)\right|+$ $\left|F\left(C_{0}\right)\right|+\left|F\left(D_{0}\right)\right| \leq\left|F\left(X^{0}\right)\right|=\xi$. So $X^{0}=B_{0} \cup C_{0} \cup D_{0}$, $\left|B_{m-1}\right|=\left|B_{0}\right|=\left|F\left(B_{0}\right)\right|,\left|C_{m-1}\right|=\left|C_{0}\right|=\left|F\left(C_{0}\right)\right|$. In particular, $1=\left|\left\{y_{2}\right\}\right|=\left|N_{G_{1}^{0}}^{m-1}\left(y_{2}\right)\right|=\left|F\left(N_{G_{1}^{0}}^{m-1}\left(y_{2}\right)\right)\right|$ because $y_{2} \in B_{m-1}$ and $g=2 m+2$. Considering the edge $x y_{2}$ similarly, we have $1=\left|\left\{y_{1}\right\}\right|=\left|N_{G_{1}^{0}}^{m-1}\left(y_{1}\right)\right|=\left|F\left(N_{G_{1}^{0}}^{m-1}\left(y_{1}\right)\right)\right|$, that is, $1=\left|\left\{y_{1}\right\}\right|=\left|D_{0}\right|=\left|F\left(D_{0}\right)\right|$. Since $\left|B_{m-1}\right|=$ $\left|B_{0}\right|=\left|F\left(B_{0}\right)\right|$ and $\left|\left\{y_{1}\right\}\right|=\left|D_{0}\right|=\left|F\left(D_{0}\right)\right|$, we have $\left|N_{G_{1}^{m-1}}(x)\right|=\left|B_{m-1} \cup\left\{y_{1}\right\}\right|=\left|B_{0} \cup D_{0}\right|=\left|N_{G_{1}^{0}}^{m}(x)\right|$ and $\left|N_{G_{1}^{0}}^{m-1}(y)\right|=\left|N_{G_{2}^{0}}^{m}(y)\right|=1$ for any $y \in N_{G}(x)$. The proof is complete.

For an edge $e=x y \in E\left(G_{1}^{m}\right)$, we shall use the following notation in Lemma 2.6, Lemma 2.7, and in the next section: $A_{m}=N_{G_{1}^{m}}(x)-\{y\}, B_{m}=N_{G_{1}^{m}}(y)-\{x\}, C_{m-1}=$ $N_{G_{1}^{m-1}}(x), D_{m-1}=N_{G_{1}^{m-1}}(y)$, and $A_{m-1}=N_{G_{1}^{m-1}}\left(A_{m}\right)$,
$B_{m-1}=N_{G_{1}^{m-1}}\left(B_{m}\right)$. Furthermore, $A_{0}=N_{G_{1}^{0}}^{m-1}\left(A_{m-1}\right), B_{0}=$ $N_{G_{1}^{0}}^{m-1}\left(B_{m-1}\right), C_{0}=N_{G_{1}^{0}}^{m-1}\left(C_{m-1}\right), D_{0}=N_{G_{1}^{0}}^{m-1}\left(D_{m-1}\right)$.

Lemma 2.6. Let $G$ be a $\lambda^{\prime}$-optimal graph with $\delta \geq 3$. Then there is no $\lambda^{\prime}-$ cut such that $1 \leq m \leq(g-5) / 2$.

Proof. Suppose, on the contrary, there exists a $\lambda^{\prime}-c u t$ $F=\left[X^{0}, Y^{0}\right]$ such that $1 \leq m \leq(g-5) / 2$. By Lemma 2.2 , we can choose an edge $e=x y \in E\left(G_{1}^{m}\right)$. Since $m \leq$ $(g-5) / 2$, i.e., $g \geq 2 m+5$, we have that $A_{0}, B_{0}, C_{0}, D_{0}$ are pairwise disjoint subsets of $X^{0}$ and $\left|A_{m}\right| \leq\left|A_{0}\right|,\left|B_{m}\right| \leq$ $\left|B_{0}\right|,\left|C_{m-1}\right| \leq\left|C_{0}\right|,\left|D_{m-1}\right| \leq\left|D_{0}\right|$. So we have

$$
\begin{aligned}
\xi \leq d(e) & =\left|A_{m}\right|+\left|B_{m}\right|+\left|C_{m-1}\right|+\left|D_{m-1}\right| \\
& \leq\left|A_{0}\right|+\left|B_{0}\right|+\left|C_{0}\right|+\left|D_{0}\right| \\
& \leq\left|F\left(A_{0}\right)\right|+\left|F\left(B_{0}\right)\right|+\left|F\left(C_{0}\right)\right|+\left|F\left(D_{0}\right)\right| \\
& \leq\left|F\left(X^{0}\right)\right|=|F|=\lambda^{\prime}=\xi .
\end{aligned}
$$

Therefore, $X^{0}=A_{0} \cup B_{0} \cup C_{0} \cup D_{0}$, and $d_{G_{1}^{m-2}}(u)=1$ if $m \geq 2$ and $d_{G_{2}^{0}}(u)=1$ if $m=1$ for any vertex $u \in D_{m-1}$. Let $u \in D_{m-1}$. Suppose there exists a vertex $w \in N_{G_{1}^{m-1}}(u)$. Since $N_{G_{1}^{0}}^{m-1}(w) \subseteq X^{0}$, we can find a cycle of length at most $2 m+3$ through $y, u, w$, contradicting $g \geq 2 m+5$. Hence $d_{G_{1}^{m-1}}(u)=$ 0 . By $d(u) \geq \delta \geq 3$, we have $I_{m}=N_{G_{1}^{m}}(u)-\{y\} \neq \varnothing$ and hence we can pick a vertex $v \in I_{m}$. Since $g \geq 2 m+5$ and $X^{0}=A_{0} \cup B_{0} \cup C_{0} \cup D_{0}$, it follows that $N_{G_{1}^{m-1}}(v)-$ $\{u\}=\emptyset$. Set $H_{m}=N_{G_{1}^{m}}\left(I_{m}\right), H_{0}=N_{G_{1}^{0}}^{m}\left(H_{m}\right)$. Since $\delta \geq$ 3 and $g \geq 2 m+5>4$, we have $\left|H_{m}\right| \geq 2\left|I_{m}\right|>\left|I_{m}\right|$. Clearly, $\left|H_{m}\right| \leq\left|H_{0}\right|$ and $H_{0} \subseteq A_{0}$. Considering the edge $y u$ similarly, we have $\left|A_{0}\right|+\left|B_{0}\right|+\left|C_{0}\right|+\left|D_{0}\right|=\xi \leq d(y u) \leq$ $\left|B_{0}\right|+\left|C_{0}\right|+\left|D_{0}\right|+\left|I_{m}\right|<\left|B_{0}\right|+\left|C_{0}\right|+\left|D_{0}\right|+\left|H_{m}\right| \leq$ $\left|B_{0}\right|+\left|C_{0}\right|+\left|D_{0}\right|+\left|A_{0}\right|$, a contradiction completing the proof.

Lemma 2.7. Let $G$ be a $\lambda^{\prime}$-optimal graph with $\delta \geq 3$ and let $F=\left[X^{0}, Y^{0}\right]$ be a $\lambda^{\prime}$-cut such that $0 \leq m=(g-4) / 2$ and $\left|V\left(G_{1}\right)\right| \geq 3$. Then there exists a cycle in $G_{1}^{m}$ such that $\left|N_{G_{1}^{0}}^{m}(w)\right|=\left|N_{G_{2}^{0}}^{m+1}(w)\right|=1$ for each vertex $w$ of the cycle.

Proof. If $m=0$, then $\left|V\left(G_{1}\right)\right|=\left|X^{0}\right| \geq 3$. This lemma follows immediately from Lemma 2.1. Therefore, we may assume $m \geq 1$. By Lemma 2.2, $E\left(G_{1}^{m}\right) \neq \emptyset$. Let $e=x y$ be an arbitrary edge in $G_{1}^{m}$.

Claim 1. $A_{0}, B_{0}, C_{0}, D_{0}$ are pairwise disjoint, $X^{0}=A_{0} \cup$ $B_{0} \cup C_{0} \cup D_{0},\left|C_{m-1}\right|=\left|C_{0}\right|,\left|D_{m-1}\right|=\left|D_{0}\right|$ and $|F(u)|=1$ for every vertex $u \in X^{0}$.

Since $m=(g-4) / 2$, that is, $g=2 m+4$, it follows that $\left|A_{0}\right| \geq\left|A_{m-1}\right| \geq\left|A_{m}\right|,\left|B_{0}\right| \geq\left|B_{m-1}\right| \geq\left|B_{m}\right|$, 
$\left|C_{0}\right| \geq\left|C_{m-1}\right|,\left|D_{0}\right| \geq\left|D_{m-1}\right|$ and $A_{0}, B_{0}, C_{0}, D_{0}$ are pairwise disjoint. So we have

$$
\begin{aligned}
\xi \leq d(e) & =\left|A_{m}\right|+\left|B_{m}\right|+\left|C_{m-1}\right|+\left|D_{m-1}\right| \\
& \leq\left|A_{m-1}\right|+\left|B_{m-1}\right|+\left|C_{m-1}\right|+\left|D_{m-1}\right| \\
& \leq\left|A_{0}\right|+\left|B_{0}\right|+\left|C_{0}\right|+\left|D_{0}\right| \\
& \leq\left|F\left(A_{0}\right)\right|+\left|F\left(B_{0}\right)\right|+\left|F\left(C_{0}\right)\right|+\left|F\left(D_{0}\right)\right| \\
& \leq\left|F\left(X^{0}\right)\right|=|F|=\lambda^{\prime}=\xi .
\end{aligned}
$$

This implies that $X^{0}=A_{0} \cup B_{0} \cup C_{0} \cup D_{0},\left|C_{0}\right|=\left|C_{m-1}\right|$, $\left|D_{0}\right|=\left|D_{m-1}\right|,\left|A_{m}\right|=\left|A_{m-1}\right|=\left|A_{0}\right|,\left|B_{m}\right|=\left|B_{m-1}\right|=$ $\left|B_{0}\right|$, and $\left|F\left(A_{0}\right)\right|=\left|A_{0}\right|,\left|F\left(B_{0}\right)\right|=\left|B_{0}\right|,\left|F\left(C_{0}\right)\right|=\left|C_{0}\right|$, $\left|F\left(D_{0}\right)\right|=\left|D_{0}\right|$. Hence $|F(u)|=1$ for any $u \in X^{0}$. The proof of Claim 1 is complete.

Suppose $A_{m} \neq \emptyset$. Since $\left|A_{m}\right|=\left|A_{m-1}\right|$ and $g=2 m+4$, it follows that $\left|N_{G_{1}^{m-1}}\left(y^{\prime}\right)\right|=1$ for any $y^{\prime} \in A_{m}=N_{G_{1}^{m}}(x)-\{y\}$. Applying the same argument to $x y^{\prime}$ for any $y^{\prime} \in A_{m}$, we have $\left|N_{G_{1}^{m-1}}\left(y^{\prime \prime}\right)\right|=1$ for any $y^{\prime \prime} \in N_{G_{1}^{m}}(x)-\left\{y^{\prime}\right\}$. In particular, $\left|D_{m-1}\right|=1$ because $y \in N_{G_{1}^{m}}(x)-\left\{y^{\prime}\right\}$. Combining this with $d(y) \geq 3$, we have $B_{m} \neq \emptyset$. Similarly, if $B_{m} \neq \emptyset$ then $\left|C_{m-1}\right|=1$ and $A_{m} \neq \emptyset$. Therefore, we have the following Claims 2 and 3 .

Claim 2. If $A_{m} \neq \emptyset$, then $\left|C_{0}\right|=\left|C_{m-1}\right|=1,\left|D_{0}\right|=$ $\left|D_{m-1}\right|=1$.

Claim 3. $A_{m}=\emptyset \Leftrightarrow B_{m}=\emptyset$.

Claim 4. There exists a vertex $z \in X^{m}$ such that $d_{G_{1}^{m}}(z) \geq 2$.

For any edge $x y \in E\left(G_{1}^{m}\right)$, if $A_{m} \neq \emptyset$, then Claim 4 follows. Suppose $A_{m}=\emptyset$. By Claim $3, B_{m}=\emptyset$. By Claim 1, we have $X^{0}=C_{0} \cup D_{0}, C_{0} \cap D_{0}=\varnothing,\left|C_{m-1}\right|=\left|C_{0}\right|$, $\left|D_{m-1}\right|=\left|D_{0}\right|$, and $|F(u)|=1$ for any $u \in X^{0}$. Let $w \in$ $C_{m-1}$. Suppose $N_{G_{1}^{m-1}}(w) \neq \emptyset$ and let $w^{\prime} \in N_{G_{1}^{m-1}}(w)$. Since $N_{G_{1}^{0}}^{m-1}\left(w^{\prime}\right) \subseteq X^{0}=C_{0} \cup D_{0}$, we can find a cycle of length at most $2 m+2$, a contradiction implying $d_{G_{1}^{m-1}}(w)=0$. By Claim 1, we have $\left|N_{G_{1}^{m-2}}(w)\right|=1$ if $m \geq 2$ and $\left|N_{G_{2}^{0}}(w)\right|=1$ if $m=1$. Thus $\left|N_{G_{1}^{m}}(w)\right| \geq 2$, which implies that there exists a vertex $z \in N_{G_{1}^{m}}(w)-\{x\}$. We have $N_{G_{1}^{m-1}}(z)=\{w\}$. Otherwise, there exists a vertex $v \in N_{G_{1}^{m-1}}(z)-\{w\}$. Since $N_{G_{1}^{0}}^{m-1}(v) \subseteq X^{0}=C_{0} \cup D_{0}$, we can find a cycle of length at most $2 m+3$ through $x, w, z, v$, which contradicts $g=2 m+4$. Thus, $d_{G_{1}^{m}}(z)=d_{G}(z)-\left|N_{G_{1}^{m-1}}(z)\right| \geq 2$, as claimed.

Now we begin to prove the lemma. By Claims 3 and 4 , we can choose an edge $x y \in E\left(G_{1}^{m}\right)$ such that $A_{m} \neq \emptyset, B_{m} \neq$ $\emptyset$. Let $H$ be the component of $G_{1}^{m}$ containing the edge $x y$. We will show $\delta(H) \geq 2$. Otherwise, there exists $z \in V(H)$ such that $d_{H}(z) \leq 1$. Clearly, $z \notin\{x, y\}$ and $d_{H}(z)=1$. Let $N_{H}(z)=\{w\}$. By the connectivity of $H, N_{H}(w)-\{z\} \neq \emptyset$ and hence $N_{G_{1}^{m}}(w)-\{z\} \neq \varnothing$. Considering the edge $w z$, it follows that $N_{G_{1}^{m}}(z)-\{w\} \neq \emptyset$ from Claim 3 and $N_{G_{1}^{m}}(w)-\{z\} \neq \emptyset$. So $N_{H}(z)-\{w\} \neq \emptyset$, contradicting $d_{H}(z) \leq 1$. Therefore,
$\delta(H) \geq 2$. This implies that there exists a cycle in $H$ and hence in $G_{1}^{m}$. This lemma follows from Claims 1 and 2.

\section{MAIN RESULTS}

Theorem 3.1. Let $G$ be a graph with $\delta \geq 3, D \leq g-2$ which is not isomorphic to the graph $W$ shown in Figure 1 . If $F=\left[V\left(G_{1}\right), V\left(G_{2}\right)\right]$ is a $\lambda^{\prime}$-cut such that each vertex of $G_{1}$ is incident with at least one edge of $F$, then either $\left|V\left(G_{1}\right)\right|=2$ or $\left|V\left(G_{2}\right)\right|=2$.

Proof. By Theorem B, $G$ is a $\lambda^{\prime}$-optimal graph. It is easy to verify that every complete graph of order at least 4 is super $-\lambda^{\prime}$. Hence it suffices to prove the case $D \geq 2$. Since $D \leq g-2$, it follows that $g \geq 4$.

Since $F=\left[V\left(G_{1}\right), V\left(G_{2}\right)\right]$ is a $\lambda^{\prime}$-cut such that each vertex of $G_{1}$ is incident with at least one edge of $F$, it follows that $V\left(G_{1}\right)=X^{0}$. Assume by way of contradiction that $\left|V\left(G_{1}\right)\right| \geq$ 3 and $\left|V\left(G_{2}\right)\right| \geq 3$. By Lemma 2.1, $|F(x)|=1$ for each vertex $x \in V\left(G_{1}\right)$ and $G_{1}$ is a complete bipartite graph, say $G_{1}=\left(S_{1}, S_{2}\right)$, where $\left|S_{1}\right| \geq 2,\left|S_{2}\right| \geq 2$. This shows that there exists a cycle of length 4 in $G_{1}$. So $g=4$ and hence $D=2$. Let $U_{1}=N_{G_{2}}\left(S_{1}\right), U_{2}=N_{G_{2}}\left(S_{2}\right)$. It is easy to see that $U_{1} \cap U_{2}=\emptyset, Y^{0}=U_{1} \cup U_{2}$.

Suppose $V\left(G_{2}\right)-Y_{0} \neq \varnothing$ and let $w$ be an arbitrary vertex in $V\left(G_{2}\right)-Y^{0}$. We consider the distance between $w$ and every vertex in $V\left(G_{1}\right)$. Since $D=2$ and $|F(u)|=1$ for each $u \in V\left(G_{1}\right)$, the vertex $w$ is adjacent to every vertex in $U_{i}, i=1,2$. Combining this with $g=4$, it follows that $E\left(G\left[Y^{0}\right]\right)=\emptyset$. Suppose $\left|U_{1}\right|>1$ and let $y_{1}, y_{2}$ be two distinct vertices in $U_{1}$. We have that the distance between $y_{1}$ and each vertex in $N_{G_{1}}\left(y_{2}\right)$ is at least 3 , contradicting $D=2$. Therefore $\left|U_{1}\right|=1$. We also have $\left|U_{2}\right|=1$ similarly. In this case, since $d(w) \geq 3$, there is a vertex $w^{\prime} \in V\left(G_{2}\right)-Y^{0}$ such that $w w^{\prime} \in E(G)$. Since $w$ is arbitrary, the vertex $w^{\prime}$ is adjacent to every vertex in $U_{1}$ and $U_{2}$, too. It follows that there exists a cycle of length 3 through $w, w^{\prime}$, a contradiction. Hence $V\left(G_{2}\right)=Y^{0}$. By Lemma 2.1, $|F(y)|=1$ for each vertex $y \in V\left(G_{2}\right)$ and $G_{2}$ is a complete bipartite graph, say $G_{2}=\left(T_{1}, T_{2}\right)$, where $\left|T_{1}\right| \geq 2,\left|T_{2}\right| \geq 2$.

We will show that $\left|N_{G_{2}}\left(S_{1}\right) \cap T_{1}\right| \leq 1$. Otherwise, let $y_{1}, y_{2} \in N_{G_{2}}\left(S_{1}\right) \cap T_{1}$. Then there exist $x_{1}, x_{2} \in S_{1}$ such that $x_{1} y_{1}, x_{2} y_{2} \in F$. It follows that $d\left(x_{1}, y_{2}\right) \geq 3$ from the fact that $|F(v)|=1$ for each $v \in V(G)$. This is contrary to $D=2$. Thus $\left|N_{G_{2}}\left(S_{1}\right) \cap T_{1}\right| \leq 1$. Similarly, we conclude $\mid N_{G_{2}}\left(S_{1}\right) \cap$ $T_{2} \mid \leq 1$. Moreover, since $\left|S_{1}\right| \geq 2$, we have $\mid N_{G_{2}}\left(S_{1}\right) \cap$ $T_{i} \mid=1(i=1,2)$ and hence $\left|S_{1}\right|=2$. Furthermore, we have $\left|N_{G_{2}}\left(S_{2}\right) \cap T_{i}\right|=1(i=1,2)$ and $\left|S_{2}\right|=2$ similarly. It follows that $G$ is isomorphic to the graph $W$, contradicting the hypothesis. The proof is complete.

The following corollary shows that Conjecture A is true for graphs with $D=2$.

Corollary 3.1. Let $G$ be a graph with $\delta \geq 3, D=2$ and $g \geq 4$. Then $G$ is super $-\lambda^{\prime}$ if and only if $G$ is not isomorphic to the graph $W$ shown in Figure 1. 


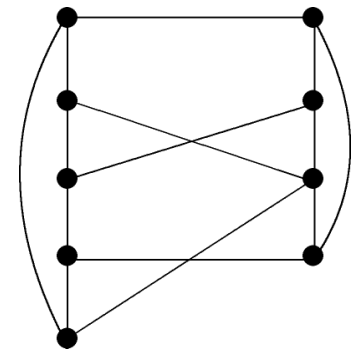

FIG. 3. A graph with $\delta=3, D=2$, and $g=4$.

Proof. The necessity is trivial. We shall prove the sufficiency below. Let $G$ be not isomorphic to $W$ and let $F=$ $\left[V\left(G_{1}\right), V\left(G_{2}\right)\right]$ be a $\lambda^{\prime}-c u t$. If there exist two vertices $x \in V\left(G_{1}\right), y \in V\left(G_{2}\right)$ such that $F(x)=\emptyset$ and $F(y)=\emptyset$, then $d(x, y) \geq 3$, contradicting the hypothesis $D=2$. So we may assume, without loss of generality, that each vertex in $V\left(G_{1}\right)$ is incident with at least one edge in $F$. Since $D=2$ and $g \geq 4$, we have $D \leq g-2$. It follows from Theorem 3.1 that either $\left|V\left(G_{1}\right)\right|=2$ or $\left|V\left(G_{2}\right)\right|=2$ and so $G$ is super $-\lambda^{\prime}$.

It is easy to verify that if $G$ is a graph with order $v \geq 8$, girth $g \geq 4$, and $d(x)+d(y) \geq v-1$ for every pair of non-adjacent vertices $x, y$, then $D=2$ and $\delta \geq 3$. Therefore, Corollary 3.1 implies the following theorem due to Fan:

Theorem D ([12]). Suppose a graph $G$ of order $v>7$ contains no cycle of length 3. If $d(x)+d(y) \geq v-1$ for every pair of non-adjacent vertices $x, y$, then $G$ is super $-\lambda^{\prime}$.

Consider the graph shown in Figure 3. By Corollary 3.1, we know that the graph is super $-\lambda^{\prime}$, but Theorem D does not apply to the graph. So Corollary 3.1 improves Theorem D in this sense.

Without loss of generality, we shall assume $m \leq n$ in this section.

Lemma 3.1. Let $G$ be a graph with $\delta \geq 3, D \leq g-2$. Then $m \leq(g-3) / 2$ for any $\lambda^{\prime}$-cut $F=\left[X^{0}, Y^{0}\right]$. Furthermore, if $F=\left[X^{0}, Y^{0}\right]$ is a $\lambda^{\prime}$-cut such that $m=(g-3) / 2$, then $m=n=(g-3) / 2, g=2 m+3, D=2 m+1$ and $d(x, y)=$ $2 m+1$ for any $x \in X^{m}, y \in Y^{n}$.

Proof. Since $m \leq n$, it follows that $D \geq d(x, y) \geq$ $m+n+1 \geq 2 m+1$ for any $x \in X^{m}, y \in Y^{n}$. So $g \geq D+2 \geq$ $2 m+3$, which implies that $m \leq(g-3) / 2$. If $m=(g-3) / 2$, then $m \leq n \leq d(x, y)-m-1 \leq D-m-1 \leq g-3-m=m$. Consequently, $m=n=(g-3) / 2, g=2 m+3$ and $D=$ $d(x, y)=2 m+1$. The proof is complete.

Lemma 3.2. Let $G$ be a graph with $\delta \geq 3, D \leq g-2$, and let $F=\left[X^{0}, Y^{0}\right]$ be a $\lambda^{\prime}$-cut such that $1 \leq m=(g-3) / 2$. If there exists a vertex $x \in X^{m}$ such that $\left|N_{G_{2}^{0}}^{m+1}(x)\right| \leq 2$, then $d_{G_{2}^{n}}(v) \leq 1$ for every vertex $v \in Y^{n}$.
Proof. Suppose, on the contrary, there exists a vertex $v_{2} \in Y^{n}$ such that $d_{G_{2}^{n}}\left(v_{2}\right) \geq 2$. Then we can find a path $P=v_{1} v_{2} v_{3}$ in $G_{2}^{n}$. Let $P_{1}, P_{2}$, and $P_{3}$ be the shortest paths from $x$ to $v_{1}, v_{2}$, and $v_{3}$, respectively. By Lemma 3.1, we have $n=m, D=2 m+1$, and hence the length of $P_{i}(i=$ $1,2,3)$ is $2 m+1$. It follows that $V\left(P_{i}\right) \cap N_{G_{2}^{0}}^{m+1}(x) \neq \emptyset$. Since $\left|N_{G_{2}^{0}}^{m+1}(x)\right| \leq 2$, there exist two paths in $\left\{P_{1}, P_{2}, P_{3}\right\}$, say $P_{j}, P_{k}$, such that $V\left(P_{j}\right) \cap V\left(P_{k}\right) \cap N_{G_{2}^{0}}^{m+1}(x) \neq \emptyset$. Let $V\left(P_{j}\right) \cap V\left(P_{k}\right) \cap N_{G_{2}^{0}}^{m+1}(x)=\{y\}$. Clearly, there exists a cycle of length at most $2 m+2$ through $y, v_{j}, v_{k}$, which contradicts the fact that $g=2 m+3$. The proof is complete.

Lemma 3.3. Let $G$ be a graph with $\delta \geq 3, D \leq g-2$ and let $F=\left[X^{0}, Y^{0}\right]$ be a $\lambda^{\prime}$-cut such that $1 \leq m=(g-3) / 2$. Then $g=2 m+3, D=2 m+1$ and $\xi(G)=4$.

Proof. By Theorem B, $G$ is a $\lambda^{\prime}$-optimal graph. By Lemma 3.1, we get $n=m=(g-3) / 2, g=2 m+3$, and $D=2 m+1$. It follows that $E\left(G_{1}^{m}\right) \neq \emptyset, E\left(G_{2}^{n}\right) \neq \emptyset$ from Lemma 2.2 and $1 \leq n=m=(g-3) / 2$. Let $u v \in E\left(G_{2}^{n}\right)$. For any edge $x y \in E\left(G_{1}^{m}\right)$, we have the following.

Claim 1. $\left|F\left(A_{0}\right)\right| \geq 2\left|A_{m}\right|$.

We need only prove Claim 1 for the case $A_{m} \neq \emptyset$. Let $z$ be an arbitrary vertex in $A_{m}$. By Lemma 3.1, $d(z, u)=$ $d(z, v)=2 m+1$. Let $P_{1}, P_{2}$ be shortest paths from $z$ to $u$ and $v$, respectively. Set $\left\{e_{i}\right\}=E\left(P_{i}\right) \cap F$ for each $i \in\{1,2\}$. Clearly, $e_{1}, e_{2} \in F\left(A_{0}\right)$. We have $e_{1} \neq e_{2}$. Indeed, if $e_{1}=e_{2}$, then there exists a cycle of length at most $2 m+1$ through $u, v$ in $G_{2}$, which contradicts $g=2 m+3$. Suppose $z^{\prime}$ is another vertex in $A_{m}$ and let $P_{1}^{\prime}$ and $P_{2}^{\prime}$ be shortest paths from $z^{\prime}$ to $u$ and $v$, respectively. Set $\left\{e_{i}^{\prime}\right\}=E\left(P_{i}^{\prime}\right) \cap F$ for each $i \in\{1,2\}$. Similarly, $e_{1}^{\prime} \neq e_{2}^{\prime}$. If there exist $i, j \in\{1,2\}$ such that $e_{i}=e_{j}^{\prime}$, then since $z, z^{\prime} \in A_{m}$, there is a cycle of length at most $2 m+2$ through $z, x, z^{\prime}$ in $G_{1}$, a contradiction. Therefore, $e_{1}, e_{2}, e_{1}^{\prime}, e_{2}^{\prime}$ are four distinct edges in $F\left(A_{0}\right)$. Claim 1 follows.

Claim 2. $A_{0}, C_{0}, D_{0}$ are pairwise disjoint, $X^{0}=A_{0} \cup$ $C_{0} \cup D_{0}, A_{0}=B_{0},\left|F\left(A_{0}\right)\right|=2\left|A_{m}\right|=2\left|B_{m}\right|,\left|F\left(C_{0}\right)\right|=$ $\left|C_{0}\right|=\left|C_{m-1}\right|$ and $\left|F\left(D_{0}\right)\right|=\left|D_{0}\right|=\left|D_{m-1}\right|$.

Without loss of generality, assume $\left|A_{m}\right| \geq\left|B_{m}\right|$. Since $g=2 m+3$, it follows that $\left|C_{0}\right| \geq\left|C_{m-1}\right|,\left|D_{0}\right| \geq\left|D_{m-1}\right|$ and $A_{0}, C_{0}, D_{0}$ are pairwise disjoint. Combining this with Claim 1, we have

$$
\begin{aligned}
\xi \leq d(x y) & =\left|A_{m}\right|+\left|B_{m}\right|+\left|C_{m-1}\right|+\left|D_{m-1}\right| \\
& \leq 2\left|A_{m}\right|+\left|C_{m-1}\right|+\left|D_{m-1}\right| \\
& \leq 2\left|A_{m}\right|+\left|C_{0}\right|+\left|D_{0}\right| \\
& \leq\left|F\left(A_{0}\right)\right|+\left|F\left(C_{0}\right)\right|+\left|F\left(D_{0}\right)\right| \\
& \leq\left|F\left(X^{0}\right)\right|=|F|=\lambda^{\prime}=\xi .
\end{aligned}
$$


This implies that $X^{0}=A_{0} \cup C_{0} \cup D_{0},\left|A_{m}\right|=\left|B_{m}\right|,\left|F\left(A_{0}\right)\right|=$ $2\left|A_{m}\right|,\left|F\left(C_{0}\right)\right|=\left|C_{0}\right|=\left|C_{m-1}\right|$ and $\left|F\left(D_{0}\right)\right|=\left|D_{0}\right|=$ $\left|D_{m-1}\right|$. Since $\left|A_{m}\right|=\left|B_{m}\right|$, we can get $X^{0}=B_{0} \cup C_{0} \cup D_{0}$ similarly. It follows that $A_{0}=B_{0}$. The proof of Claim 2 is complete.

Claim 3. If $A_{m} \neq \emptyset$, then $\left|N_{G_{1}^{m-1}}(z)\right|=\left|N_{G_{1}^{0}}^{m}(z)\right|=2$ for any $z \in A_{m} \cup B_{m} \cup\{x, y\}$ and $|F(w)|=1$ for any $w \in X^{0}$.

For any vertex $y^{\prime} \in A_{m}$, let $A_{m}^{\prime}=A_{m} \cup\{y\}-\left\{y^{\prime}\right\}$ and consider the edge $x y^{\prime}$. It is easy to see that $N_{G_{1}^{0}}^{m}\left(A_{m}^{\prime}\right)=$ $A_{0} \cup N_{G_{1}^{0}}^{m}(y)-N_{G_{1}^{0}}^{m}\left(y^{\prime}\right)$. Similar to the proof of $\left|F\left(A_{0}\right)\right|=$ $2\left|A_{m}\right|$ in Claim 2, we have $\left|F\left(A_{0} \cup N_{G_{1}^{0}}^{m}(y)-N_{G_{1}^{0}}^{m}\left(y^{\prime}\right)\right)\right|=$ $2\left|A_{m} \cup\{y\}-\left\{y^{\prime}\right\}\right|=2\left|A_{m}\right|=\left|F\left(A_{0}\right)\right|$, which implies $\left|F\left(N_{G_{1}^{0}}^{m}\left(y^{\prime}\right)\right)\right|=\left|F\left(N_{G_{1}^{0}}^{m}(y)\right)\right|=\left|F\left(D_{0}\right)\right|=\left|D_{0}\right|$. Since $2\left|A_{m}\right|=\left|F\left(A_{0}\right)\right|=\left|F\left(\cup_{y^{\prime} \in A_{m}} N_{G_{1}^{0}}^{m}\left(y^{\prime}\right)\right)\right|=\mid \cup_{y^{\prime} \in A_{m}}$ $\left(F\left(N_{G_{1}^{0}}^{m}\left(y^{\prime}\right)\right)\right)|=| F\left(D_{0}\right)|| A_{m}|=| D_{0}|| A_{m} \mid$, we get $\left|F\left(D_{0}\right)\right|=$ $\left|D_{0}\right|=\left|D_{m-1}\right|=2$, and hence $|F(z)|=1$ for any $z \in D_{0}$. Considering edges $y x, x y^{\prime}, y x^{\prime}$ for every $y^{\prime} \in A_{m}, x^{\prime} \in B_{m}$ similarly, we can conclude $\left|N_{G_{1}^{m-1}}(z)\right|=\left|N_{G_{1}^{0}}^{m}(z)\right|=2$ for any vertex $z \in\{x, y\} \cup A_{m} \cup B_{m}$ and $|F(w)|=1$ for any $w \in X^{0}$. The proof of Claim 3 is complete.

Claim 4. $\quad d_{G_{1}^{m}}(x) \leq 1$ for every vertex $x \in X^{m}$.

Suppose, on the contrary, that there exists a vertex $x$ in $X^{m}$ such that $d_{G_{1}^{m}}(x) \geq 2$. Then there exists $y \in N_{G_{1}^{m}}(x)$ such $\left|A_{m}\right|=k>0$ for the edge $x y$. By Claim 3 and $g=2 m+3$, we have $\left|A_{0}\right|=2\left|A_{m}\right|$ and $\left|C_{0}\right|=\left|D_{0}\right|=2$. Combining this with Claim 2, $\xi=2\left|A_{m}\right|+\left|C_{0}\right|+\left|D_{0}\right|=\left|A_{0}\right|+\left|C_{0}\right|+\left|D_{0}\right|=$ $2 k+4$ and hence $\left|X^{0}\right|=2 k+4$. Since $\left|F\left(D_{0}\right)\right|=2$, it follows that $\left|N_{G_{2}^{0}}^{m+1}(y)\right| \leq 2$. By Lemma 3.2, $d_{G_{2}^{n}}(w) \leq 1$ for every vertex $w \in Y^{n}$.

For the above edge $u v$, let $U_{n-1}=N_{G_{2}^{n-1}}(u), V_{n-1}=$ $N_{G_{2}^{n-1}}(v), U_{0}=N_{G_{2}^{0}}^{n}(u), V_{0}=N_{G_{2}^{0}}^{n}(v)$. Since $d_{G_{2}^{n}}(w) \leq 1$ for every vertex $w \in Y^{n}, N_{G_{2}^{n}}(u)-\{v\}=N_{G_{2}^{n}}(v)-\{u\}=\emptyset$. Similar to the proof of Claim 2, we have $Y^{0}=U_{0} \cup V_{0}$, $\left|U_{n-1}\right|=\left|U_{0}\right|=\left|F\left(U_{0}\right)\right|,\left|V_{n-1}\right|=\left|V_{0}\right|=\left|F\left(V_{0}\right)\right|$, and hence $|F(w)|=1$ for any vertex $w \in Y^{0}$. It follows that $\left|U_{0}\right|+\left|V_{0}\right|=\left|Y^{0}\right|=\left|X^{0}\right|=2 k+4$. Since the distance between $u$ (respectively, $v$ ) and each vertex in $\{x, y\} \cup A_{m}$ is $2 m+1$, we have $\left|U_{0}\right| \geq k+2$ (respectively, $\left|V_{0}\right| \geq k+2$ ) and hence $\left|U_{0}\right|=\left|V_{0}\right|=k+2$ and $d(v)=k+3$. Let $P$ be a shortest $(y, v)$-path. Then there is exactly one vertex in $V(P) \cap Y^{n-1}$, say $z$. The fact that $d(z v)=d(z)+d(v)-2 \geq$ $\xi=2 k+4$ implies that $d(z) \geq 2 k+6-d(v)=k+3$. Since $N_{G_{2}^{0}}^{n-1}\left(N_{G_{2}^{n-1}}(z)\right) \subseteq Y^{0}=U_{0} \cup V_{0}$ and $g=2 m+3$, we have $N_{G^{n-1}}(z)=\emptyset$. Since $\left|V_{n-1}\right|=\left|V_{0}\right|=\left|F\left(V_{0}\right)\right|$, we have $\left|N_{G^{n-2}}^{2}(z)\right|=1$ if $n \geq 2$ and $\left|N_{G_{1}^{0}}(z)\right|=1$ if $n=1$. It follows that $\left|N_{G_{2}^{n}}(z)\right|=d(z)-1 \geq k+2 \geq 3$. So there exist two distinct vertices $v_{1}, v_{2} \in N_{G_{2}^{n}}(z)-\{v\}$. Since $\delta \geq 3$ and $d_{G_{2}^{n}}(w) \leq 1$ for every vertex $w \in Y^{n}$, it follows that $H_{i}=N_{G_{2}^{n-1}}\left(v_{i}\right)-\{z\} \neq \emptyset, i=1$, 2. Since $g=2 m+3, N_{G_{2}^{0}}^{n-1}\left(H_{i}\right) \subseteq U_{0}$. By Lemma 3.1, $d(x, u)=$ $d(x, v)=2 m+1$. Combining this with Claim 3, we conclude that there is exactly one vertex in $U_{0}$, say $u_{0}$, such that $d\left(x, u_{0}\right)=m+1$. By Lemma 3.1, $d\left(v_{i}, x\right)=2 m+1$ which implies $\left\{u_{0}\right\} \subseteq N_{G_{2}^{0}}^{n}\left(v_{1}\right) \cap N_{G_{2}^{0}}^{n}\left(v_{2}\right)$. Then there exists a cycle of length at most $2 m+2$ through $u_{0}, v_{1}, z, v_{2}$, a contradiction completing the proof of Claim 4.

Now we begin to prove this lemma. By Claim $4, d_{G_{1}^{m}}(x) \leq$ 1 for every $x \in X^{m}$. So for any edge $x y \in E\left(G_{1}^{m}\right), A_{m}=B_{m}=$ $\emptyset$. It follows from Claim 2 that $X^{0}=C_{0} \cup D_{0}$ and $|F(z)|=1$ for any $z \in X^{0}$. Analogously, we also have that $N_{G_{2}^{n}}(u)-\{v\}=$ $N_{G_{2}^{n}}(v)-\{u\}=\emptyset$ and hence $\left|U_{n-1}\right|=\left|U_{0}\right|,\left|V_{n-1}\right|=\left|V_{0}\right|$, $Y^{0}=U_{0} \cup V_{0},|F(w)|=1$ for any vertex $w \in Y^{0}$, where $U_{n-1}, V_{n-1}, U_{0}, V_{0}$ are defined as above. (Note $m=n$.)

Let $P$ be a shortest $(y, v)$-path and let $J_{m}=N_{G_{1}^{m}}(z)-\{y\}$, where $z \in V(P) \cap X^{m-1}$. The fact that $d(z y)=d(z)+d(y)-$ $2 \geq \xi=\left|C_{0}\right|+\left|D_{0}\right|$ implies that $d(z) \geq\left|C_{0}\right|+\left|D_{0}\right|+2-$ $d(y)=\left|C_{0}\right|+1$. Since $N_{G_{1}^{0}}^{m-1}\left(N_{G_{1}^{m-1}}(z)\right) \subseteq X^{0}=C_{0} \cup D_{0}$ and $g=2 m+3$, we have $N_{G_{1}^{m-1}}(z)=\emptyset$. Since $\left|D_{m-1}\right|=\left|D_{0}\right|=$ $\left|F\left(D_{0}\right)\right|$, we have $\left|N_{G_{1}^{m-2}}(z)\right|=1$ if $m \geq 2$, and $\left|N_{G_{2}^{0}}(z)\right|=1$ if $m=1$. It follows that $\left|J_{m}\right|=d(z)-2 \geq\left|C_{0}\right|-1$. Since $d_{G_{1}^{m}}(x) \leq 1$ for every $x \in X^{m}$, we have $N_{G_{1}^{m-1}}\left(y^{\prime}\right)-\{z\} \neq \emptyset$ for any $y^{\prime} \in J_{m}$ and hence $N_{G_{1}^{m-1}}\left(J_{m}\right)-\{z\} \neq \emptyset$. Let $J_{m-1}=$ $N_{G_{1}^{m-1}}\left(J_{m}\right)-\{z\}$ and $J_{0}=N_{G_{1}^{0}}^{m-1}\left(J_{m-1}\right)$. Since $g=2 m+3$, we have $J_{0} \cap D_{0}=\emptyset$ and so $J_{0} \subseteq C_{0}$. By Lemma 3.1, the distance between $u$ and each vertex in $J_{m}$ is $2 m+1$, which implies that there exist at least $\left(\left|C_{0}\right|-1\right)$ vertices in $C_{0}$ such that each of them is adjacent to some vertex in $U_{0}$. Combining this with $d(x, v)=2 m+1$ and $|F(w)|=1$ for any $w \in X^{0} \cup Y^{0}$, there exists exactly one vertex in $C_{0}$ such that it is adjacent to some vertex in $V_{0}$. That is $\left|\left[C_{0}, V_{0}\right]\right|=1$. Similarly, we have $\left|\left[C_{0}, U_{0}\right]\right|=\left|\left[D_{0}, U_{0}\right]\right|=\left|\left[D_{0}, V_{0}\right]\right|=1$. Therefore, $|F|=4$ and hence $\xi=4$. The proof is complete.

Lemma 3.4. Let $G$ be a graph with $\delta \geq 3, D \leq g-2$. If $F=\left[X^{0}, Y^{0}\right]$ is a $\lambda^{\prime}$-cut of $G$ such that $1 \leq m \leq(g-4) / 2$, then $D=2 m+2, g=2 m+4$.

Proof. By Theorem B, $G$ is a $\lambda^{\prime}$-optimal graph. By Lemma 2.6, we have $m=(g-4) / 2$, that is, $g=2 m+4$. Suppose that $n>m$ and let $x \in X^{m}, u \in Y^{n}$. Since $2 m+2=g-2 \geq D \geq d(x, u) \geq m+n+1>2 m+1$, we have $D=2 m+2$. Suppose $n=m$. It is easy to verify that $\left|V\left(G_{1}\right)\right| \geq 3$ because $m \geq 1$. Therefore, by Lemma 2.7, there exists a cycle in $G_{1}^{m}$ such that $\left|N_{G_{1}^{0}}^{m}(w)\right|=\left|N_{G_{2}^{0}}^{m+1}(w)\right|=1$ for every vertex $w$ of this cycle. In particular, there exists an edge $u v \in E\left(G_{1}^{m}\right)$ such that $\left|N_{G_{1}^{0}}^{m}(u)\right|=\left|N_{G_{2}^{0}}^{m+1}(u)\right|=1$ and $\left|N_{G_{1}^{0}}^{m}(v)\right|=\left|N_{G_{2}^{0}}^{m+1}(v)\right|=1$. Since $n=m=(g-4) / 2$, we can apply Lemma 2.7 to $G_{2}$, too. It follows that there exists a vertex $y \in Y^{n}$ such that $\left|N_{G_{2}^{0}}^{n}(y)\right|=1$. Without loss of generality, assume $N_{G_{2}^{0}}^{m+1}(u) \neq N_{G_{2}^{0}}^{n}(y)$. Then $d(u, y) \geq 2 m+2$. Combining this with $D \leq g-2=2 m+2$, we conclude that $D=2 m+2$. The proof is complete.

Theorem 3.2. Let $G$ be a graph with $\delta \geq 3, D \leq g-2$. If $G$ is not super- $\lambda^{\prime}$, then there exists a non-negative integer 
$k$ such that either $g=2 k+3, D=2 k+1, \xi(G)=4$ or $g=2 k+4, D=2 k+2$.

Proof. By Theorem B, $G$ is a $\lambda^{\prime}$-optimal graph. Since $G$ is not super- $\lambda^{\prime}$, there exists a $\lambda^{\prime}$-cut $F=\left[V\left(G_{1}\right), V\left(G_{2}\right)\right]=$ $\left[X^{0}, Y^{0}\right]$ such that $\left|V\left(G_{1}\right)\right| \geq 3,\left|V\left(G_{2}\right)\right| \geq 3$. If $G$ is isomorphic to the graph $W$ shown in Figure 1 , then $g=4, D=2$. If $G$ is not isomorphic to the graph $W$, then by Theorem 3.1, $V\left(G_{1}\right)-X^{0} \neq \varnothing$, that is, $m \geq 1$. By Lemmas 2.6 and 3.1, $(g-4) / 2 \leq m \leq(g-3) / 2$. This theorem follows from Lemmas 3.3 and 3.4 .

Now we present an immediate consequence of Theorem 3.2.

Corollary 3.2. Let $G$ be a graph with $\delta \geq 3, D \leq g-3$. Then $G$ is super $-\lambda^{\prime}$.

If $D \leq g-3$, then since Diam $_{1} \leq$ Diam $_{0}=D$, we have $\operatorname{Diam}_{1} \leq g-3$. Clearly, the converse is not true. The following theorems improve Corollary 3.2 in this sense.

Theorem 3.3. Let $G$ be a connected graph with $\delta \geq 3$ and odd girth $g \geq 5$. Then, (1) $G$ is super $-\lambda^{\prime}$ if Diam $1 \leq g-3$; (2) $G$ is super $-\lambda^{\prime}$ if both Diam $1=g-2$ and Diam Dia $_{1} \leq g-5$.

Proof. Recall a connected graph $G$ is $\lambda^{\prime}$-connected if $\delta \geq$ 3. By Theorem C, $G$ is a $\lambda^{\prime}$-optimal graph if Diam $_{1} \leq g-3$, or both Diam $_{1}=g-2$ and Diam $2 \leq g-5$. Suppose, on the contrary, that $G$ is not super $-\lambda^{\prime}$. Then there exists a $\lambda^{\prime}-c u t$ $F=\left[V\left(G_{1}\right), V\left(G_{2}\right)\right]=\left[X^{0}, Y^{0}\right]$ such that $\left|V\left(G_{1}\right)\right| \geq 3$ and $\left|V\left(G_{2}\right)\right| \geq 3$. Without loss of generality, assume $m \leq n$. If $m=0$, then $\left|X^{0}\right|=\left|V\left(G_{1}\right)\right| \geq 3$. By Lemma 2.1, we can find a cycle of length 4 in $G_{1}$, which contradicts $g \geq 5$. Therefore, $m \geq 1$. Combining Lemma 2.6 with the fact that $g$ is odd, we have $m \geq(g-3) / 2$.

Let $G$ be a connected graph with $\delta \geq 3$, odd girth $g \geq 5$ and Diam $_{1} \leq g-3$. Suppose $m \geq(g-1) / 2$. Considering the distance between an edge in $\left[X^{m}, X^{m-1}\right]$ and an edge in $\left[Y^{n}, Y^{n-1}\right]$, we have $\operatorname{Diam}_{1} \geq(m-1)+(n-1)+1 \geq$ $g-2$, a contradiction. Suppose $m=(g-3) / 2$ and $n>$ $(g-3) / 2$. By Lemma 2.2, there exists an edge in $G_{1}^{m}$. Thus, $\operatorname{Diam}_{1} \geq m+(n-1)+1 \geq g-2$, a contradiction. Suppose $m=n=(g-3) / 2$. In this case, by Lemma 2.2, there exist $e_{1} \in E\left(G_{1}^{m}\right), e_{2} \in E\left(G_{2}^{n}\right)$. Thus, Diam $_{1} \geq m+n+1=g-2$, a contradiction again. Thus, the proof of (1) is complete.

Let $G$ be a connected graph with $\delta \geq 3$, odd girth $g \geq 5$, $\operatorname{Diam}_{1}=g-2$ and Diam $_{2} \leq g-5$. Assume $m=(g-3) / 2$. If $n=(g-3) / 2$, or $n=(g-1) / 2$ and $G_{2}^{n}$ contains no edge, then by Lemmas 2.3 and 2.4, there are two cycles such that the distance between them is at least $g-4$, contradicting Diam $_{2} \leq g-5$. If $n=(g-1) / 2$ and $G_{2}^{n}$ contains edges, or $n>(g-1) / 2$, then by Lemma 2.2, there exist two edges such that the distance between them is at least $g-1$, contradicting $\operatorname{Diam}_{1}=g-2$. Therefore, $m \geq(g-1) / 2$. If $m=(g-$ $1) / 2, n=(g-1) / 2$ and neither $G_{1}^{m}$ nor $G_{2}^{n}$ contains edges, then we can find two cycles such that the distance between them is at least $g-4$ from Lemma 2.4, a contradiction. For the remaining cases, it is easy to find two edges such that the distance between them is at least $g-1$. This is a contradiction. The proof of (2) is complete.

Theorem 3.4. Let $G$ be a connected graph with $\delta \geq 3$, even girth $g \geq 4$, Diam $_{1} \leq g-3$, and Diam $2 \leq g-4$. Then $G$ is super $-\lambda^{\prime}$.

Proof. Clearly, $G$ is $\lambda^{\prime}$-connected. By Theorem C, $G$ is a $\lambda^{\prime}$-optimal graph. Suppose, on the contrary, that $G$ is not super $-\lambda^{\prime}$. Then there exists a $\lambda^{\prime}-$ cut $F=\left[V\left(G_{1}\right), V\left(G_{2}\right)\right]=$ $\left[X^{0}, Y^{0}\right]$ such that $\left|V\left(G_{1}\right)\right| \geq 3$ and $\left|V\left(G_{2}\right)\right| \geq 3$. Without loss of generality, assume $m \leq n$. By Lemma 2.6, we have $m \geq(g-4) / 2$ or $m=0$. If $m=0$, then by Lemma 2.1, we can find a cycle of length 4 in $G_{1}$ and hence $g=4$. In this case, $m=0=(g-4) / 2$.

Assume $0 \leq m=(g-4) / 2$. By Lemma 2.7, there exists a cycle $C$ in $\bar{G}_{1}^{m}$ such that $\left|N_{G_{1}^{0}}^{m}(w)\right|=\left|N_{G_{2}^{0}}^{m+1}(w)\right|=1$ for each vertex $w$ of $C$. If $n=(g-4) / 2$, then, by Lemma 2.7, $G_{2}^{n}$ also contains cycles. This is contrary to Diam $_{2} \leq g-4$. If $n>(g-2) / 2$, or $n=(g-2) / 2$ and $G_{2}^{n}$ has edges, then there exist two edges such that the distance between them is at least $g-2$, a contradiction. If $n=(g-2) / 2$, $G_{2}^{n}$ contains no edge, but $G_{2}\left[Y^{n} \cup Y^{n-1}\right]$ has cycles, then the distance between a cycle in $G_{2}\left[Y^{n} \cup Y^{n-1}\right]$ and $C$ is at least $g-3$, a contradiction. Suppose $n=(g-2) / 2, G_{2}^{n}$ contains no edge and $G_{2}\left[Y^{n} \cup Y^{n-1}\right]$ contains no cycle. For any edge $u v$ of the cycle $C$, we may assume, by the choice of $C$, that $N_{G_{2}^{0}}^{m+1}(u)=\left\{u_{0}\right\}, N_{G_{2}^{0}}^{m+1}(v)=\left\{v_{0}\right\}$. By Lemma 2.5, there exists a vertex $x$ in $Y^{n}$ such that $\left|N_{G_{2}^{0}}^{n-1}(y)\right|=1$ for any $y \in N_{G_{2}^{n-1}}(x)$. Since $G_{2}^{n}$ contains no edge and $\delta \geq 3$, we have $\left|N_{G_{2}^{n-1}}(x)\right| \geq 3$. By Lemma $2.5,\left|N_{G_{2}^{0}}^{n}(x)\right| \geq 3$ and hence there exists a vertex $y \in N_{G_{2}^{n-1}}(x)$ such that $N_{G_{2}^{0}}^{n-1}(y) \cap\left\{u_{0}, v_{0}\right\}=$ $\emptyset$. Thus, $d\left(u_{0}, y\right)>n-1, d\left(v_{0}, y\right)>n-1$. This implies that $d(u, y)>n+m=g-3, d(v, y)>n+m=g-3$, and hence the distance between $x y$ and $u v$ is at least $g-2$, a contradiction again. Thus, $m \geq(g-2) / 2$ (this implies $m \geq 1$ ).

Suppose $m=n=(g-2) / 2$ and neither $G_{1}^{m}$ nor $G_{2}^{n}$ contains edges. If both $G_{1}\left[X^{m} \cup X^{m-1}\right]$ and $G_{2}\left[Y^{n} \cup Y^{n-1}\right]$ have cycles, then we can get a contradiction to $\operatorname{Diam}_{2} \leq g-4$. Without loss of generality, we assume $G_{1}\left[X^{m} \cup X^{m-1}\right]$ contains no cycle. Let $x$ be the vertex in $X^{m}$ which is given in Lemma 2.5 and let $y \in N_{G_{1}^{m-1}}(x)$. By Lemma 2.5, we may assume $N_{G_{2}^{0}}^{m}(y)=\left\{y_{0}\right\}$. Choose $u \in Y^{n}$ and let $v_{1}, v_{2} \in N_{G_{2}^{n-1}}(u)$. If $y_{0} \in N_{G_{2}^{0}}^{n-1}\left(v_{1}\right) \cap N_{G_{2}^{0}}^{n-1}\left(v_{2}\right)$, then there exists a cycle of length at most $2 n$, which implies that $n \geq g / 2$, a contradiction. Therefore, we may assume, without loss of generality, that $y_{0} \notin N_{G_{2}^{0}}^{n-1}\left(v_{1}\right)$. Then $d\left(y_{0}, v_{1}\right)>$ $n-1$ and hence $d\left(y, v_{1}\right)>n-1+m=g-3$. So the distance between the edge $x y$ and the edge $u v$ is at least $g-2$, a contradiction. For the remaining cases, it is easy to find two edges such that the distance between 
them is at least $g-2$. This is a contradiction. The proof is complete.

\section{CONCLUSION}

The super restricted edge connectivity is an essential parameter of an interconnection network. In this paper, we study the super restricted edge connectivity of graphs with small diameter or conditional diameter. First, we point out that a conjecture on super restricted edge connectivity is true for graphs with diameter 2 though it is false in general. Second, we give sufficient conditions for a graph to be super restricted edge-connected in terms of its diameter and its girth. Third, we present sufficient conditions on the conditional diameter (instead of the diameter itself) and the girth for super restricted edge-connected graphs. Finally, we expect that the methods used in this paper can be generalized to discuss other parameters, such as $k(>2)$ restricted edge connectivity.

\section{REFERENCES}

[1] C. Balbuena, A. Carmona, J. Fábrega, and M.A. Fiol, On the connectivity and the conditional diameter of graphs and digraphs, Networks 28 (1996), 97-105.

[2] C. Balbuena, A. Carmona, J. Fábrega, and M.A. Fiol, Superconnectivity of bipartite digraphs and graphs, Discr Math 197-198 (1999), 61-75.

[3] C. Balbuena, M. Cera, A. Diánez, P. García-Vázquez, and X. Marcote, Sufficient conditions for $\lambda^{\prime}$-optimality of graphs with small conditional diameter, Inform Process Lett 95 (2005), 429-434.

[4] C. Balbuena, P. García-Vázquez, and X. Marcote, Sufficient conditions for $\lambda^{\prime}$-optimality in graphs with girth $g$, J Graph Theor 52 (2006), 73-86.

[5] M.O. Ball, Complexity of network reliability computation, Networks 10 (1980), 153-165.

[6] D. Bauer, F. Boesch, C. Suffel, and R. Tindell, "Connectivity extremal problems and the design of reliable probabilistic networks", The theory and application of graphs, Wiley, New York, 1981, pp. 89-98.

[7] D. Bauer, F. Boesch, C. Suffel, and R. Tindell, Combinatorial optimization problem in analysis and design of probabilistic networks, Networks 15 (1985), 257-271.

[8] J.A. Bondy and U.S.R. Murty, Graph theory with applications, The Macmillan Press Ltd, New York, 1976.

[9] A.H. Esfahanian and S.L. Hakimi, On computing a conditional edge-connectivity of a graph, Inform Process Lett 27 (1988), 195-199.

[10] J. Fábrega and M.A. Fiol, Maximally connected digraphs, J Graph Theor 13 (1989), 657-668.

[11] J. Fábrega and M.A. Fiol, On the extraconnectivity of graphs, Discr Math 155 (1996), 49-57.

[12] Y.M. Fan, A sufficient condition for super restricted edgeconnectivity, J Guangxi Univ Technol (Chin Ed) 14 (2003), 66-68.

[13] A. Hellwig and L. Volkmann, Sufficient conditions for $\lambda^{\prime}$ optimality in graphs of diameter 2, Discr Math 283 (2004), 113-120.

[14] A. Hellwig and L. Volkmann, Sufficient conditions for graphs to be $\lambda^{\prime}$-optimal, super-edge-connected, and maximally edgeconnected, J Graph Theor 48 (2005), 228-246.

[15] Q.L. Li and Q. Li, Reliability analysis of circulant graphs, Networks 31 (1998), 61-65.

[16] J.S. Provan and M.O. Ball, The complexity of counting cuts and of computing the probability that a graph is connected, SIAM J Comput 12 (1983), 777-788.

[17] T. Soneoka, H. Nakada, M. Imase, and C. Peyrat, Sufficient conditions for maximally connected dense graphs, Discr Math 63 (1987), 53-66.

[18] M. Wang and Q. Li, Conditional edge connectivity properties, reliability comparisons and transitivity of graphs, Discr Math 258 (2002), 205-214.

[19] Y.Q. Wang and Q. Li, A sufficient condition for the equality between the restricted edge-connectivity and minimum edgedegree of graphs, Appl Math J Chin Univ Ser A (Chin Ed) 16 (2001), 269-275. 